\title{
Measuring the Pollutants in a System of Three Interconnecting Lakes by the Semianalytical Method
}

\author{
Indranil Ghosh $\mathbb{D}^{1},{ }^{1}$ M. S. H. Chowdhury $\mathbb{D}^{1},{ }^{1}$ Suazlan Mt Aznam $\mathbb{D}^{1},{ }^{1}$ and M. M. Rashid $\mathbb{D}^{2}$ \\ ${ }^{1}$ Department of Science in Engineering, Faculty of Engineering, International Islamic University Malaysia, Jalan Gombak, \\ 53100 Kuala Lumpur, Malaysia \\ ${ }^{2}$ Department of Mechatronics Engineering, Faculty of Engineering, International Islamic University Malaysia, Jalan Gombak, \\ 53100 Kuala Lumpur, Malaysia
}

Correspondence should be addressed to M. S. H. Chowdhury; sazzadbd@iium.edu.my

Received 8 October 2020; Revised 4 April 2021; Accepted 8 May 2021; Published 15 June 2021

Academic Editor: Bruno Carpentieri

Copyright (c) 2021 Indranil Ghosh et al. This is an open access article distributed under the Creative Commons Attribution License, which permits unrestricted use, distribution, and reproduction in any medium, provided the original work is properly cited.

Pollution has become an intense danger to our environment. The lake pollution model is formulated into the three-dimensional system of differential equations with three instances of input. In the present study, the new iterative method (NIM) was applied to the lake pollution model with three cases called impulse input, step input, and sinusoidal input for a longer time span. The main feature of the NIM is that the procedure is very simple, and it does not need to calculate any special type of polynomial or multipliers such as Adomian polynomials and Lagrange's multipliers. Comparisons with the Adomian decomposition method $(\mathrm{ADM})$ and the well-known purely numerical fourth-order Runge-Kutta method (RK4) suggest that the NIM is a powerful alternative for differential equations providing more realistic series solutions that converge very rapidly in real physical problems.

\section{Introduction}

Over the years, differential equations are employed by researchers that care about what is going on in the surroundings, for example, one can see the following ref. [1-10]. Therefore, differential equations play a prominent role to solve physical problems using numerical or analytical methods. There has recently been much attention devoted to the search for better and more efficient solution methods for determining a solution, approximate or exact, analytical, or numerical, to physical models. Finding exact or approximate solutions of these nonlinear equations is interesting and important. Several semianalytic methods including the Adomian decomposition method (ADM) [11], homotopy perturbation method (HPM) [12], and variational iteration method (VIM) [13] have been used for solving mathematical models of differential equations.

Finding accurate and analytical procedures for solving lake pollution model has been a vital research undertaking. The system of lake pollution models has been solved first time by Biazar et al. [14] using a semianalytic technique called the Adomian decomposition method (ADM). So often, it is very hard to ascertain the Adomian polynomials involved with ADM. To overcome the shortcomings of $\mathrm{ADM}$, many analytical techniques have been successfully implemented by various groups of researchers. In particular, the lake pollution system was solved by Ganji et al. [15] via homotopy-perturbation method (HPM). Recently, Ahmed and Khan [16] have investigated dynamics of system of polluted lakes through the numerical procedure fractional derivative approach. Numerous authors used some of the approximate numerical techniques to obtain approximate analytical solutions such as the Bessel matrix method [17], Haar wavelet collocation method [18], Differential Transform method (DTM) [19], Laplace-Padé Differential transform method (LPDTM) [20], and variational iteration method (VIM) [21].

Another prominent semianalytical technique which has been demonstrated to be a lot more straightforward and efficient than the abovementioned methods is called the new iterative method (NIM), first proposed by Varsha Daftardar-Gejji and Jafari [22] . Very recently, NIM was 
employed for solving Fokker-Planck equations [23], chemical kinetic equations [24], and Klein-Gordon equations [25]. Some other applications of the new iterative method (NIM) on nonlinear problems can be found in [26-29]. In this paper, the NIM motivates us to solve the lake pollution systems.

\section{Mathematical Modeling of the Lake Pollution System}

Pollutants have different kinds of characteristic property, and nonpolar pollutants never mix with lake water, whereas polar one makes a reaction with lake water; as a result, mild to severe contamination takes place [30]. Figure 1 represents the three lakes with interconnecting passage with each other. We contemplate every lake to be a big compartment and interconnecting passage as pipes between the compartments. In the figure, each arrow pointed out the flow direction of the pollutant through the channels and $q(t)$ represents the rate of the pollutant that enters the lake per unit time. The volume of water $v_{i}$ in each lake remains constants, and amount of the pollutant is denoted by $z_{i}(t)$, constantly distributed in each lake at any time $t \geq 0$ where $i=1,2,3$. Here, the concentration of the contaminant in each lake is represented by $c_{i}(t)=z_{i}(t) / v_{i}$. We consider the constant flow rate is $F_{j i}$ and the motion of the flow is in the direction from lake $i$ to lake $j$, and there is no reverse flow. The flux rate is symbolized by $R_{j i}(t)$ and can be defined as $R_{j i}(t)=F_{j i} c_{i}(t)=F_{j i}\left(z_{i}(t) / v_{i}\right)$.

Now, the rate of change of contaminant $=$ input rate in every lake is the output rate in every lake. For the volume of every lake to stay steady, the stream rate into every lake must adjust the progression of the lake. So, we acquire the accompanying conditions between (1), (2), and (3):

$$
\begin{gathered}
\text { Lake I. } F_{13}=F_{31}+F_{21} \\
\text { Lake II. } F_{32}=F_{21} \\
\text { Lake III. } F_{31}+F_{32}=F_{13} \\
\text { The following system of differential equations yielded as } \\
\text { the lake pollution model: } \\
\qquad \frac{d z_{1}}{d t}=\frac{F_{13}}{v_{3}} z_{3}(t)+q(t)-\frac{F_{31}}{v_{1}} z_{1}(t)-\frac{F_{21}}{v_{1}} z_{1}(t) \\
\frac{d z_{2}}{d t}=\frac{F_{21}}{v_{1}} z_{1}(t)-\frac{F_{32}}{v_{2}} z_{2}(t) \\
\frac{d z_{3}}{d t}=\frac{F_{31}}{v_{1}} z_{1}(t)+\frac{F_{32}}{v_{2}} z_{2}(t)-\frac{F_{13}}{v_{3}} z_{3}(t)
\end{gathered}
$$

With initial conditions,

$$
z_{1}(0)=z_{2}(0)=z_{3}(0)=0 .
$$

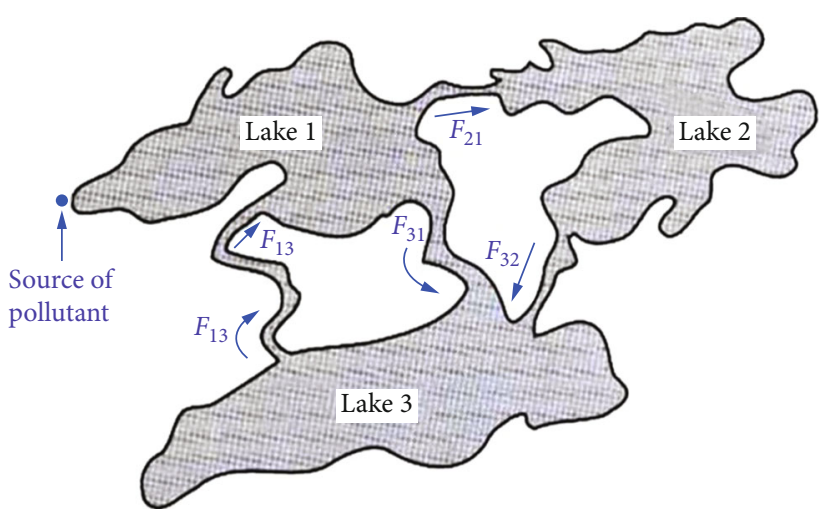

FIGURE 1: Interconnecting channels of the system of three lakes [14].

The parametric values are $v_{1}=2900 \mathrm{mi}^{3}, v_{2}=850 \mathrm{mi}^{3}$, $v_{3}=1180 \mathrm{mi}^{3}$,

$$
\begin{aligned}
& F_{21}=18 \mathrm{mi}^{3} / \text { year }, \\
& F_{32}=18 \mathrm{mi}^{3} / \text { year }, \\
& F_{31}=20 \mathrm{mi}^{3} / \text { year }, \\
& F_{13}=38 \mathrm{mi}^{3} / \text { year. }
\end{aligned}
$$

\section{Analysis of the Method}

3.1. New Iterative Method (NIM). In this section, we have discussed the new iterative method (NIM) $[31,32]$ as follows. Let us consider the general equation:

$$
u(y)=N(u(y))+f(y)
$$

where $N$ is a nonlinear operator from a Banach space $\mathscr{B}$ $\longrightarrow \mathscr{B}$, and $f$ is a known function.

Let $y=\left(y_{1}, y_{2}, \ldots . ., y_{n}\right)$ be a solution of $u$ for equation (7) having the series form.

$$
u(y)=\sum_{i=0}^{\infty} u_{i}(y)
$$

The nonlinear operator $N$ can be written as

$$
N\left(\sum_{i=0}^{\infty} u_{i}\right)=N\left(u_{0}\right)+\sum_{i=1}^{\infty}\left\{N\left(\sum_{j=0}^{i} u_{j}\right)-N\left(\sum_{j=0}^{i-1} u_{j}\right)\right\} .
$$

Then, series solution becomes

$$
\sum_{i=1}^{\infty} u_{i}=f+N\left(u_{0}\right)+\sum_{i=1}^{\infty}\left\{N\left(\sum_{j=0}^{i} u_{j}\right)-N\left(\sum_{j=0}^{i-1} u_{j}\right)\right\} .
$$

We define the consecutive relation:

$$
\begin{aligned}
& u_{0}=f, \\
& u_{1}=N\left(u_{0}\right),
\end{aligned}
$$




$$
\begin{aligned}
u_{2} & =N\left(u_{0}+u_{1}\right)-N\left(u_{0}\right), \\
u_{m+1} & =N\left(u_{0}+\cdots+u_{m}\right)-N\left(u_{0}+\cdots+u_{m-1}\right), m=1,2, \cdots
\end{aligned}
$$

Therefore,

$$
u=f+\sum_{i=1}^{\infty} u_{i}
$$

3.2. Stability Analysis. Let $X, Y$ be Banach spaces and $F: X \longrightarrow Y$ a map. $L(X, Y)$ denotes the set of all linear maps from $X$ to $Y . L(X, Y)$ is also a Banach space.

Definition 1 (see $[33,34]$ ). $F$ is said to be Fréchet differentiable at $x \in X$ if there exists a continuous linear map $A: X$ $\longrightarrow Y$ such that

$$
F(x+h)-F(x)=A h+w(x, h),
$$

where $\lim _{\|h\| \longrightarrow 0}(\|w(x, h)\| /\|h\|)=0 . A$ is called the Fréchet derivative of $F$ at $x$ and is also denoted by $F^{\prime}(x)$. Its value at $h$ is denoted by $F^{\prime}(x)(h)$. Note that $F^{\prime}$ is a linear map from $X$ to $L(X, Y)$.

Definition 2 (see $[33,34]) . F$ is said to be twice differentiable if the map $F^{\prime}: X \longrightarrow L(X, Y)$ is Fréchet differentiable. The second derivative of $F$ is denoted by $F^{\prime \prime}$ and is a linear map from $X$ to $L(X, L(X, Y))$ that is isomorphic to $L(X \times X, Y)$.

Theorem 3 (see $[33,34])$. The map $F^{\prime \prime}(x) \in L\left(X^{2}, Y\right)$ is symmetric, that $F^{\prime \prime}(x)\left(x_{1}, x_{2}\right)=F^{\prime \prime}(x)\left(x_{2}, x_{1}\right)$, where $x_{1}, x_{2} \in X$.

In this manner, $F^{(3)}(x), F^{(4)}(x), \cdots$ is inductively defined, and $F^{(n)}(x) \in L\left(X^{n}, Y\right)$ is multilinear and symmetric map.

Theorem 4 (see $[33,34])$. [Taylor's theorem]). Suppose that $F \in C^{n}(U)$, where $U$ is an open subset of $X$ containing the line segment from $x_{0}$ to $h$, and then

$$
\begin{aligned}
F\left(x_{0}+h\right)= & F\left(x_{0}\right)+F^{\prime}\left(x_{0}\right)(h)+\frac{1}{2 !} F^{\prime \prime}\left(x_{0}\right)(h, h)+\cdots \\
& +\frac{1}{(n-1) !} F^{(n-1)}\left(x_{0}\right) \underbrace{(h, \cdots h)}_{n-1 \text { times }} \\
& +\frac{1}{(n-1) !} \int_{0}^{1}(1-t)^{n-1} F^{(n)} \\
& \cdot\left(x_{0}+t h\right) \underbrace{(h, \cdots h)}_{n \text { times }} d t+q(x), \\
= & \sum_{k=0}^{n} \frac{1}{(n-1) !} F^{(n-1)}\left(x_{0}\right) \underbrace{(h, \cdots h)}_{k \text { times }},
\end{aligned}
$$

where $q(x)$ is such that $\|q(x)\|=o\left(\|x\|^{n}\right)$.
Since $F^{(k)}(x)$ is symmetric, we denote $\underbrace{(h, \cdots h)}_{k \text { times }}$ by $h^{k}$.

Using Theorem 4,

$$
\begin{aligned}
G_{1}= & N\left(u_{0}+u_{1}\right)-N\left(u_{0}\right)=\left(N\left(u_{0}\right)\right. \\
& \left.+N^{\prime}\left(u_{0}\right) u_{1}+N^{\prime \prime}\left(u_{0}\right) \frac{u_{1}^{2}}{2 !}+\cdots\right)-N\left(u_{0}\right) \\
= & \sum_{k=1}^{\infty} N^{(k)}\left(u_{0}\right) \frac{u_{1}^{k}}{k !}
\end{aligned}
$$

$$
\begin{aligned}
G_{2} & =N\left(u_{0}+u_{1}+u_{2}\right)-N\left(u_{0}+u_{1}\right) \\
& =N^{\prime}\left(u_{0}+u_{1}\right) u_{2}+N^{\prime \prime}\left(u_{0}+u_{1}\right) \frac{u_{2}^{2}}{2 !}+\cdots \\
& =\sum_{j=1}^{\infty}\left[\sum_{i=0}^{\infty} N^{(i+j)}\left(u_{0}\right) \frac{u_{1}^{i}}{i !}\right] \frac{u_{2}^{j}}{j !}
\end{aligned}
$$

$$
G_{3}=\sum_{i_{3}=1}^{\infty} \sum_{i_{2}=0}^{\infty} \sum_{i_{1}=0}^{\infty} N^{\left(i_{1}+i_{2}+i_{3}\right)}\left(u_{0}\right) \frac{u_{3}^{i_{3}}}{i_{3} !} \frac{u_{2}^{i_{2}}}{i_{2} !} \frac{u_{1}^{i_{1}}}{i_{1} !} .
$$

In general,

$$
G_{n}=\sum_{i_{n}=1}^{\infty} \sum_{i_{n-1}=0}^{\infty} \ldots \sum_{i_{1}=0}^{\infty}\left[N\left(\sum_{k=1}^{n} i_{k}\right)\left(u_{0}\right)\left(\prod_{j=1}^{n} \frac{u_{j}^{i_{j}}}{i_{j} !}\right)\right] .
$$

In view of (9)-(15) and (18)-(21),

$$
\begin{aligned}
N(u)= & G_{0}+G_{1}+G_{2}+G_{3}+\cdots=N\left(u_{0}\right) \\
& +\sum_{k=1}^{\infty} N^{(k)}\left(u_{0}\right) \frac{u_{1}^{k}}{k !}+\sum_{j=1}^{\infty}\left[\sum_{i=0}^{\infty} N^{(i+j)}\left(u_{0}\right) \frac{u_{1}^{i}}{i !}\right] \frac{u_{2}^{j}}{j !} \\
& +\sum_{i_{3}=1}^{\infty} \sum_{i_{2}=0}^{\infty} \sum_{i_{1}=0}^{\infty} N^{\left(i_{1}+i_{2}+i_{3}\right)}\left(u_{0}\right) \frac{u_{3}^{i_{3}}}{i_{3} !} \frac{u_{2}^{i_{2}}}{i_{2} !} \frac{u_{1}^{i_{1}}}{i_{1} !}+\cdots \\
= & N\left(u_{0}\right)+N^{\prime}\left(u_{0}\right)\left[u_{1}+u_{2}+u_{3}+\cdots\right] \\
& +\frac{N^{\prime \prime}\left(u_{0}\right)}{2 !}\left[u_{1}+u_{2}+u_{3}+\cdots\right]^{2} \\
& +\frac{N^{(3)}\left(u_{0}\right)}{3 !}\left[u_{1}+u_{2}+u_{3}+\cdots\right]^{3}+\cdots
\end{aligned}
$$

Equation (22) is Taylor series expansion of $N(u)$ around $u_{0}$. Thus, NIM is equivalent to Taylor series expansion around $u_{0}$. 
3.3. Error Bound. The $n^{\text {th }}$ degree NIM polynomial at $u=u_{0}$ is

$$
\begin{aligned}
N_{n}(u)= & N\left(u_{0}\right)+N^{\prime}\left(u_{0}\right)\left[u_{1}+u_{2}+u_{3}+\cdots\right] \\
& +\frac{N^{\prime \prime}\left(u_{0}\right)}{2 !}\left[u_{1}+u_{2}+u_{3}+\cdots\right]^{2} \\
& +\frac{N^{(3)}\left(u_{0}\right)}{3 !}\left[u_{1}+u_{2}+u_{3}+\cdots\right]^{3}+\cdots \\
& +\frac{N^{(n)}\left(u_{0}\right)}{3 !}\left[u_{1}+u_{2}+u_{3}+\cdots\right]^{n}
\end{aligned}
$$

Since the NIM approximation becomes more accurate as more terms are included, the $N_{n+1}(u)$ polynomial must be more accurate than $N_{n}(u)$.

$$
\begin{aligned}
N_{n+1}(u)= & N\left(u_{0}\right)+\frac{N^{\prime}\left(u_{0}\right)}{1 !}\left[u_{1}+u_{2}+u_{3}+\cdots\right] \\
& +\frac{N^{\prime \prime}\left(u_{0}\right)}{2 !}\left[u_{1}+u_{2}+u_{3}+\cdots\right]^{2}+\cdots \\
& +\frac{N^{(n)}\left(u_{0}\right)}{n !}\left[u_{1}+u_{2}+u_{3}+\cdots\right]^{n} \\
& +\frac{N^{(n+1)}\left(u_{0}\right)}{(n+1) !}\left[u_{1}+u_{2}+u_{3}+\cdots\right]^{n+1} \\
= & N_{n}(u)+\frac{N^{(n+1)}\left(u_{0}\right)}{(n+1) !}\left[u_{1}+u_{2}+u_{3}+\cdots\right]^{n+1}
\end{aligned}
$$

Since the difference between $N_{n}(u)$ and $N_{n+1}(u)$ is just that last term, the error of $N_{n}(u)$ can be no larger than that term. In other words, the error $E_{n}$ is

$$
E_{n}(u)=\max \left(\frac{N^{(n+1)}\left(u_{0}\right)}{(n+1) !}\left[u_{1}+u_{2}+u_{3}+\cdots\right]^{n+1}\right) .
$$

Since $u_{0}$ and $n$ are constant in this formula, terms depending only on those constants and $u$ are unaffected by the max operator and can be pulled outside:

$$
E_{n}(u)=\frac{\max \left(N^{(n+1)}\left(u_{0}\right)\right)}{(n+1) !}\left(\left[u_{1}+u_{2}+u_{3}+\cdots\right]^{n+1}\right) .
$$

The largest value obtainable by $N^{n+1}$ could not possibly exceed the maximum value of that derivative between $u_{0}$ and $u$. Call the $u$ value that provides that maximum value $\omega$ , and the error becomes

$$
E_{n}(u)=\frac{N^{(n+1)}(\omega)}{(n+1) !}\left[u_{1}+u_{2}+u_{3}+\cdots\right]^{n+1} \text { for all } \omega \in\left[u_{0}, u\right] .
$$

\subsection{Convergence Analysis}

Theorem 5. Let $\chi, \gamma$ and $u$ be the elements in a Banach Space $B$, and $N$ is nonlinear contraction from $B \longrightarrow B$ such that $\|$ $u\|=\| N(\chi)-N(\gamma)\|\leq k\| \chi-\gamma \|$, and $0<k<1$. Then, accord- ing to the principle of Banach fixed point theorem [35], we can prove that

$$
\left\|u_{m+1}\right\| \leq k^{m+1}\left\|u_{0}\right\|, \quad m=0,1,2, \cdots
$$

In view of (11)-(14), we have

$$
\begin{aligned}
u_{0} & =f \\
\left\|u_{1}\right\| & =\left\|N\left(u_{0}\right)\right\| \leq k\left\|u_{0}\right\| \Rightarrow \frac{\left\|u_{1}\right\|}{\left\|u_{0}\right\|} \leq k \\
\left\|u_{2}\right\| & =\left\|N\left(u_{0}+u_{1}\right)-N\left(u_{0}\right)\right\| \leq k\left\|u_{1}\right\| \\
& \leq k^{2}\left\|u_{0}\right\| \Rightarrow \frac{\left\|u_{2}\right\|}{\left\|u_{1}\right\|} \leq k \\
\left\|u_{3}\right\| & =\left\|N\left(u_{0}+u_{1}+u_{2}\right)-N\left(u_{0}+u_{1}\right)\right\| \\
& \leq k\left\|u_{2}\right\| \leq k^{3}\left\|u_{0}\right\| \Rightarrow \frac{\left\|u_{3}\right\|}{\left\|u_{2}\right\|} \leq k \\
\vdots & \| \\
\left\|u_{m+1}\right\| & =\left\|\left(\sum_{j=0}^{m} u_{j}\right)-N\left(\sum_{j=0}^{m-1} u_{j}\right)\right\| \leq k\left\|u_{m}\right\| \\
& \leq k^{m+1}\left\|u_{0}\right\| \Rightarrow \frac{\left\|u_{m+1}\right\|}{\left\|u_{m}\right\|} \leq k, m=0,1,2, . .
\end{aligned}
$$

According to the Banach contraction principle, these are the necessary and sufficient conditions for convergence of the solutions of the NIM procedure. Therefore, the series $\sum_{i=0}^{\infty} u_{m}$ is absolutely and uniformly convergent to the solution of equation (7).

\section{Application of NIM}

Here, we monitor the pollutants for three different types of input models. According to the values of $q(t)$, these three models are impulse input, step input, and sinusoidal input $[14,30]$;

4.1. Impulse Input. This model is considered when there is a spike in concentration of dumped contaminant. Considering the value of $q(t)$ is 100 units, the system becomes

$$
\begin{aligned}
& \frac{d z_{1}}{d t}=\frac{38}{1180} z_{3}(t)+100-\frac{38}{2900} z_{1}(t), \\
& \frac{d z_{2}}{d t}=\frac{18}{2900} z_{1}(t)-\frac{18}{850} z_{2}(t), \\
& \frac{d z_{3}}{d t}=\frac{20}{2900} z_{1}(t)+\frac{18}{850} z_{2}(t)-\frac{38}{1180} z_{3}(t),
\end{aligned}
$$

with initial conditions $z_{1}(0)=z_{2}(0)=z_{3}(0)=0$.

Integrating the system of equations from 0 to $t$ and using the initial conditions (33), we get 


$$
\begin{aligned}
& z_{1}(t)=100 t+\int_{0}^{t} \frac{19 z_{3}(t)}{590} d t-\int_{0}^{t} \frac{19 z_{1}(t)}{1450} d t \\
& z_{2}(t)=\int_{0}^{t} \frac{9 z_{1}(t)}{1450} d t-\int_{0}^{t} \frac{9 z_{2}(t)}{425} d t \\
& z_{3}(t)=\int_{0}^{t} \frac{z_{1}(t)}{145} d t+\int_{0}^{t} \frac{9 z_{2}(t)}{425} d t-\int_{0}^{t} \frac{19 z_{3}(t)}{590} d t .
\end{aligned}
$$

We let the nonlinear terms as

$$
\begin{aligned}
& N_{1}\left(z_{1}, z_{2}, z_{3}\right)=\int_{0}^{t} \frac{19 z_{3}(t)}{590} d t-\int_{0}^{t} \frac{19 z_{1}(t)}{1450} d t \\
& N_{2}\left(z_{1}, z_{2}, z_{3}\right)=\int_{0}^{t} \frac{9 z_{1}(t)}{1450} d t-\int_{0}^{t} \frac{9 z_{2}(t)}{425} d t \\
& N_{3}\left(z_{1}, z_{2}, z_{3}\right)=\int_{0}^{t} \frac{z_{1}(t)}{145} d t+\int_{0}^{t} \frac{9 z_{2}(t)}{425} d t-\int_{0}^{t} \frac{19 z_{3}(t)}{590} d t .
\end{aligned}
$$

In view of (11)-(14), we obtain first few components of the new iterative solution for the considered problem as follows:

$$
\begin{aligned}
& z_{1,0}(t)=100 t \\
& z_{2,0}(t)=0 \text {, } \\
& z_{3,0}(t)=0 \text {, } \\
& z_{1,1}(t)=N_{1}\left(z_{1,0}, z_{2,0}, z_{3,0}\right)=\int_{0}^{t} \frac{19 z_{3,0}(t)}{590} d t \\
& -\int_{0}^{t} \frac{19 z_{1,0}(t)}{1450} d t=-\frac{19 t^{2}}{29} \\
& z_{2,1}(t)=N_{2}\left(z_{1,0}, z_{2,0}, z_{3,0}\right)=\int_{0}^{t} \frac{9 z_{1,0}(t)}{1450} d t \\
& -\int_{0}^{t} \frac{9 z_{2,0}(t)}{425} d t=\frac{9 t^{2}}{29} \\
& z_{3,1}(t)=N_{3}\left(z_{1,0}, z_{2,0}, z_{3,0}\right)=\int_{0}^{t} \frac{z_{1,0}(t)}{145} d t+\int_{0}^{t} \frac{9 z_{2,0}(t)}{425} d t \\
& -\int_{0}^{t} \frac{19 z_{3,0}(t)}{590} d t=\frac{10 t^{2}}{29} \\
& z_{1,2}(t)=N_{1}\left(z_{1,0}+z_{1,1}, z_{2,0}+z_{2,1}, z_{3,0}+z_{3,1}\right)-N_{1}\left(z_{1,0}, z_{2,0}, z_{3,0}\right) \\
& =\left\{\int_{0}^{t} \frac{19\left(z_{3,0}(t)+z_{3,1}(t)\right)}{590} d t-\int_{0}^{t} \frac{19\left(z_{1,0}(t)+z_{1,1}(t)\right)}{1450} d t\right\} \\
& -\left\{\int_{0}^{t} \frac{19 z_{3,0}(t)}{590} d t-\int_{0}^{t} \frac{19 z_{1,0}(t)}{1450} d t\right\}=\frac{16283 t^{3}}{2480950}, \\
& \begin{aligned}
z_{2,2}(t)= & N_{2}\left(z_{1,0}+z_{1,1}, z_{2,0}+z_{2,1}, z_{3,0}+z_{3,1}\right)-N_{1}\left(z_{1,0}, z_{2,0}, z_{3,0}\right) \\
= & \left\{\int_{0}^{t} \frac{9\left(z_{1,0}(t)+z_{1,1}(t)\right)}{1450} d t-\int_{0}^{t} \frac{9\left(z_{2,0}(t)+z_{2,1}(t)\right)}{425} d t\right\} \\
& -\left\{\int_{0}^{t} \frac{9 z_{1,0}(t)}{1450} d t-\int_{0}^{t} \frac{9 z_{2,0}(t)}{425} d t\right\}=-\frac{507 t^{3}}{142970},
\end{aligned}
\end{aligned}
$$

$$
\begin{aligned}
z_{3,2}(t)= & N_{3}\left(z_{1,0}+z_{1,1}, z_{2,0}+z_{2,1}, z_{3,0}+z_{3,1}\right)-N_{1}\left(z_{1,0}, z_{2,0}, z_{3,0}\right) \\
= & \left\{\int_{0}^{t} \frac{\left(z_{1,0}(t)+z_{1,1}(t)\right)}{145} d t+\int_{0}^{t} \frac{9\left(z_{2,0}(t)+z_{2,1}(t)\right)}{425} d t\right. \\
& \left.-\int_{0}^{t} \frac{19\left(z_{3,0}(t)+z_{3,1}(t)\right)}{590} d t\right\}-\left\{\int_{0}^{t} \frac{z_{1,0}(t)}{145} d t\right. \\
& \left.+\int_{0}^{t} \frac{9 z_{2,0}(t)}{425} d t-\int_{0}^{t} \frac{19 z_{3,0}(t)}{590} d t\right\}=-\frac{63623 t^{3}}{21088075}
\end{aligned}
$$

and so on. In the same manner, the successive approximations can be obtained.

Therefore, the 9-iteration approximate series solutions are

$$
\begin{aligned}
z_{1}(t)= & \sum_{i=0}^{9} z_{1, i}(t)=100 t-0.6551724138 t^{2} \\
& +0.006563211673 t^{3}-0.00004578968898 t^{4} \\
& +2.284062102 \times 10^{-7} t^{5}-7.613669180 \times 10^{-10} t^{6} \\
& +9.264952117 \times 10^{-13} t^{7}+8.062395960 \times 10^{-15} t^{8} \\
& -7.447533873 \times 10^{-17} t^{9}+3.912821015 \times 10^{-19} t^{10} \\
z_{2}(t)= & \sum_{i=0}^{9} z_{2, i}(t)=0.3103448276 t^{2} \\
& -0.003546198503 t^{3}+0.00002895828605 t^{4} \\
& -1.794892311 \times 10^{-7} t^{5}+8.697736903 \times 10^{-10} t^{6} \\
& -3.306351810 \times 10^{-12} t^{7}+9.470940223 \\
& \times 10^{-15} t^{8}-1.672429216 \times 10^{-17} t^{9} \\
& -1.080992422 \times 10^{-20} t^{10}, \\
& 9 \\
z_{3}(t)= & \sum_{i=0} z_{3, i}=0.3448275862 t^{2}-0.003017013170 t^{3} \\
+ & 0.00001683140293 t^{4}-4.891697909 \times 10^{-8} t^{5} \\
& -1.084067722 \times 10^{-10} t^{6}+2.379856598 \\
& \times 10^{-12} t^{7}-1.753333618 \times 10^{-14} t^{8} \\
+ & 9.119963089 \times 10^{-17} t^{9}-3.804721773 \times 10^{-19} t^{10}
\end{aligned}
$$

It is easily observed that the obtained solution

$\left(z_{1}, z_{2}, z_{3}\right)=\left(\sum_{i=0}^{\infty} z_{1, i}, \sum_{i=0}^{\infty} z_{2, i}, \sum_{i=0}^{\infty} z_{3, i}\right)$, converges to the exact solution when

$$
\beta_{1, i}=\frac{\left\|z_{1, i+1}\right\|}{\left\|z_{1, i}\right\|}<1, \beta_{2, i}=\frac{\left\|z_{2, i+1}\right\|}{\left\|z_{2, i}\right\|}<1, \beta_{3, i}=\frac{\left\|z_{3, i+1}\right\|}{\left\|z_{3, i}\right\|}<1
$$

for $i \geq 1,0<t \leq 1$. 
Here, we can see that

$$
\begin{aligned}
& \frac{\left\|z_{1,2}\right\|}{\left\|z_{1,1}\right\|}=\frac{\left\|\left(16283 t^{3}\right) / 2480950\right\|}{\left\|\left(19 t^{2}\right) / 29\right\|}=0.0100175336<1, \\
& \frac{\left\|z_{2,2}\right\|}{\left\|z_{2,1}\right\|}=\frac{\left\|\left(507 t^{3}\right) / 142970\right\|}{\left\|\left(9 t^{2}\right) / 29\right\|}=0.01142663961<1, \\
& \frac{\left\|z_{3,2}\right\|}{\left\|z_{3,1}\right\|}=\frac{\left\|\left(63623 t^{3}\right) / 21088075\right\|}{\left\|\left(10 t^{2}\right) / 29\right\|}=0.00874933816<1 .
\end{aligned}
$$

This confirm that the NIM series solution converges to the exact solution.

4.2. Step Input. This model is considered when contaminants enter the lake continuously at steady concentration. Considering the value of $q(t)$ is $100 t$ units, the system becomes

$$
\begin{aligned}
& \frac{d z_{1}}{d t}=\frac{38}{1180} z_{3}(t)+100 t-\frac{38}{2900} z_{1}(t) \\
& \frac{d z_{2}}{d t}=\frac{18}{2900} z_{1}(t)-\frac{18}{850} z_{2}(t), \\
& \frac{d z_{3}}{d t}=\frac{20}{2900} z_{1}(t)+\frac{18}{850} z_{2}(t)-\frac{38}{1180} z_{3}(t),
\end{aligned}
$$

with initial conditions

$$
z_{1}(0)=z_{2}(0)=z_{3}(0)=0 .
$$

Integrating the system of equations from 0 to $t$ and using the initial conditions (41), we get

$$
\begin{aligned}
& z_{1}(t)=50 t^{2}+\int_{0}^{t} \frac{19 z_{3}(t)}{590} d t-\int_{0}^{t} \frac{19 z_{1}(t)}{1450} d t, \\
& z_{2}(t)=\int_{0}^{t} \frac{9 z_{1}(t)}{1450} d t-\int_{0}^{t} \frac{9 z_{2}(t)}{425} d t \\
& z_{3}(t)=\int_{0}^{t} \frac{z_{1}(t)}{145} d t+\int_{0}^{t} \frac{9 z_{2}(t)}{425} d t-\int_{0}^{t} \frac{19 z_{3}(t)}{590} d t .
\end{aligned}
$$

We let the nonlinear terms as

$$
\begin{aligned}
& N_{1}\left(z_{1}, z_{2}, z_{3}\right)=\int_{0}^{t} \frac{19 z_{3}(t)}{590} d t-\int_{0}^{t} \frac{19 z_{1}(t)}{1450} d t, \\
& N_{2}\left(z_{1}, z_{2}, z_{3}\right)=\int_{0}^{t} \frac{9 z_{1}(t)}{1450} d t-\int_{0}^{t} \frac{9 z_{2}(t)}{425} d t \\
& N_{3}\left(z_{1}, z_{2}, z_{3}\right)=\int_{0}^{t} \frac{z_{1}(t)}{145} d t+\int_{0}^{t} \frac{9 z_{2}(t)}{425} d t-\int_{0}^{t} \frac{19 z_{3}(t)}{590} d t .
\end{aligned}
$$

In view of (11)-(14), we have the following first few components of the new iterative solution for the considered problem:

$$
\begin{aligned}
& z_{1,0}(t)=50 t^{2}, \\
& z_{2,0}(t)=0 \text {, } \\
& z_{3,0}(t)=0 \text {, } \\
& z_{1,1}(t)=N_{1}\left(z_{1,0}, z_{2,0}, z_{3,0}\right)=\int_{0}^{t} \frac{19 z_{3,0}(t)}{590} d t \\
& -\int_{0}^{t} \frac{19 z_{1,0}(t)}{1450} d t=-\frac{19 t^{2}}{87}, \\
& z_{2,1}(t)=N_{2}\left(z_{1,0}, z_{2,0}, z_{3,0}\right)=\int_{0}^{t} \frac{9 z_{1,0}(t)}{1450} d t \\
& -\int_{0}^{t} \frac{9 z_{2,0}(t)}{425} d t=\frac{3 t^{2}}{29} \\
& z_{3,1}(t)=N_{3}\left(z_{1,0}, z_{2,0}, z_{3,0}\right)=\int_{0}^{t} \frac{z_{1,0}(t)}{145} d t \\
& +\int_{0}^{t} \frac{9 z_{2,0}(t)}{425} d t-\int_{0}^{t} \frac{19 z_{3,0}(t)}{590} d t=\frac{10 t^{2}}{87}, \\
& z_{1,2}(t)=N_{1}\left(z_{1,0}+z_{1,1}, z_{2,0}+z_{2,1}, z_{3,0}+z_{3,1}\right) \\
& -N_{1}\left(z_{1,0}, z_{2,0}, z_{3,0}\right)=\left\{\int_{0}^{t} \frac{19\left(z_{3,0}(t)+z_{3,1}(t)\right)}{590} d t\right. \\
& \left.-\int_{0}^{t} \frac{19\left(z_{1,0}(t)+z_{1,1}(t)\right)}{1450} d t\right\}-\left\{\int_{0}^{t} \frac{19 z_{3,0}(t)}{590} d t\right. \\
& \left.-\int_{0}^{t} \frac{19 z_{1,0}(t)}{1450} d t\right\}=\frac{16283 t^{3}}{9923800} \text {, } \\
& z_{2,2}(t)=N_{2}\left(z_{1,0}+z_{1,1}, z_{2,0}+z_{2,1}, z_{3,0}+z_{3,1}\right) \\
& -N_{1}\left(z_{1,0}, z_{2,0}, z_{3,0}\right)=\left\{\int_{0}^{t} \frac{9\left(z_{1,0}(t)+z_{1,1}(t)\right)}{1450} d t\right. \\
& \left.-\int_{0}^{t} \frac{9\left(z_{2,0}(t)+z_{2,1}(t)\right)}{425} d t\right\}-\left\{\int_{0}^{t} \frac{9 z_{1,0}(t)}{1450} d t\right. \\
& \left.-\int_{0}^{t} \frac{9 z_{2,0}(t)}{425} d t\right\}=-\frac{507 t^{3}}{571880}, \\
& z_{3,2}(t)=N_{3}\left(z_{1,0}+z_{1,1}, z_{2,0}+z_{2,1}, z_{3,0}+z_{3,1}\right)-N_{1}\left(z_{1,0}, z_{2,0}, z_{3,0}\right) \\
& =\left\{\int_{0}^{t} \frac{\left(z_{1,0}(t)+z_{1,1}(t)\right)}{145} d t+\int_{0}^{t} \frac{9\left(z_{2,0}(t)+z_{2,1}(t)\right)}{425} d t\right. \\
& \left.-\int_{0}^{t} \frac{19\left(z_{3,0}(t)+z_{3,1}(t)\right)}{590} d t\right\}-\left\{\int_{0}^{t} \frac{z_{1,0}(t)}{145} d t\right. \\
& \left.+\int_{0}^{t} \frac{9 z_{2,0}(t)}{425} d t-\int_{0}^{t} \frac{19 z_{3,0}(t)}{590} d t\right\}=-\frac{63623 t^{3}}{84352300},
\end{aligned}
$$

and so on. In the same manner, the successive components can be obtained. 
TABLE 1: Numerical comparisons between ADM and NIM for the impulse input model.

\begin{tabular}{|c|c|c|c|c|c|c|}
\hline \multirow[b]{2}{*}{$t$} & \multicolumn{3}{|c|}{ ADM [14] } & \multicolumn{3}{|c|}{ NIM } \\
\hline & $z_{1}(t)$ & $z_{2}(t)$ & $z_{3}(t)$ & $z_{1}(t)$ & $z_{2}(t)$ & $z_{3}(t)$ \\
\hline 0.01 & 0.99993 & 0.00003 & 0.00003 & 0.99993 & 0.00003 & 0.00003 \\
\hline 0.02 & 1.99974 & 0.00012 & 0.00014 & 1.99974 & 0.00012 & 0.00014 \\
\hline 0.03 & 2.99941 & 0.00028 & 0.00031 & 2.99941 & 0.00028 & 0.00031 \\
\hline 0.04 & 3.99900 & 0.00049 & 0.00055 & 3.99895 & 0.00050 & 0.00055 \\
\hline 0.05 & 4.99895 & 0.00076 & 0.00086 & 4.99836 & 0.00078 & 0.00086 \\
\hline 0.06 & 5.99836 & 0.00112 & 0.00124 & 5.99764 & 0.00112 & 0.00124 \\
\hline 0.07 & 6.99764 & 0.00152 & 0.00169 & 6.99679 & 0.00152 & 0.00169 \\
\hline 0.08 & 7.99679 & 0.00198 & 0.00221 & 7.99581 & 0.00198 & 0.00221 \\
\hline 0.09 & 8.99581 & 0.00251 & 0.00279 & 8.99469 & 0.00251 & 0.00279 \\
\hline 0.1 & 9.99345 & 0.00310 & 0.00345 & 9.99345 & 0.00310 & 0.00345 \\
\hline
\end{tabular}

TABLE 2: Differences between 9-iteration NIM with RK4 solutions in the impulse input model.

\begin{tabular}{lccc}
\hline & & $\Delta=R K 4_{0.001}-\mathrm{NIM}$ & $\Delta z_{3}(t)$ \\
\hline 2 & $\Delta z_{1}(t)$ & $1.28 e-13$ & $1.42 e-13$ \\
4 & $1.02 e-11$ & $7.62 e-13$ & $8.56 e-13$ \\
6 & $3.02 e-11$ & $1.08 e-12$ & $8.2 e-13$ \\
8 & $3.19 e-11$ & $2.39 e-12$ & $6.27 e-12$ \\
10 & $1.038 e-10$ & $2.349 e-11$ & $1.306 e-10$ \\
12 & $1.435 e-10$ & $1.763 e-10$ & $9.677 e-10$ \\
14 & $1.028 e-09$ & $9.273 e-10$ & $5.216 e-09$ \\
16 & $5.914 e-09$ & $3.946 e-09$ & $2.248 e-08$ \\
18 & $2.608 e-08$ & $1.419 e-08$ & $8.153 e-08$ \\
20 & $9.562 e-08$ & $4.472 e-08$ & $2.582 e-07$ \\
\hline
\end{tabular}
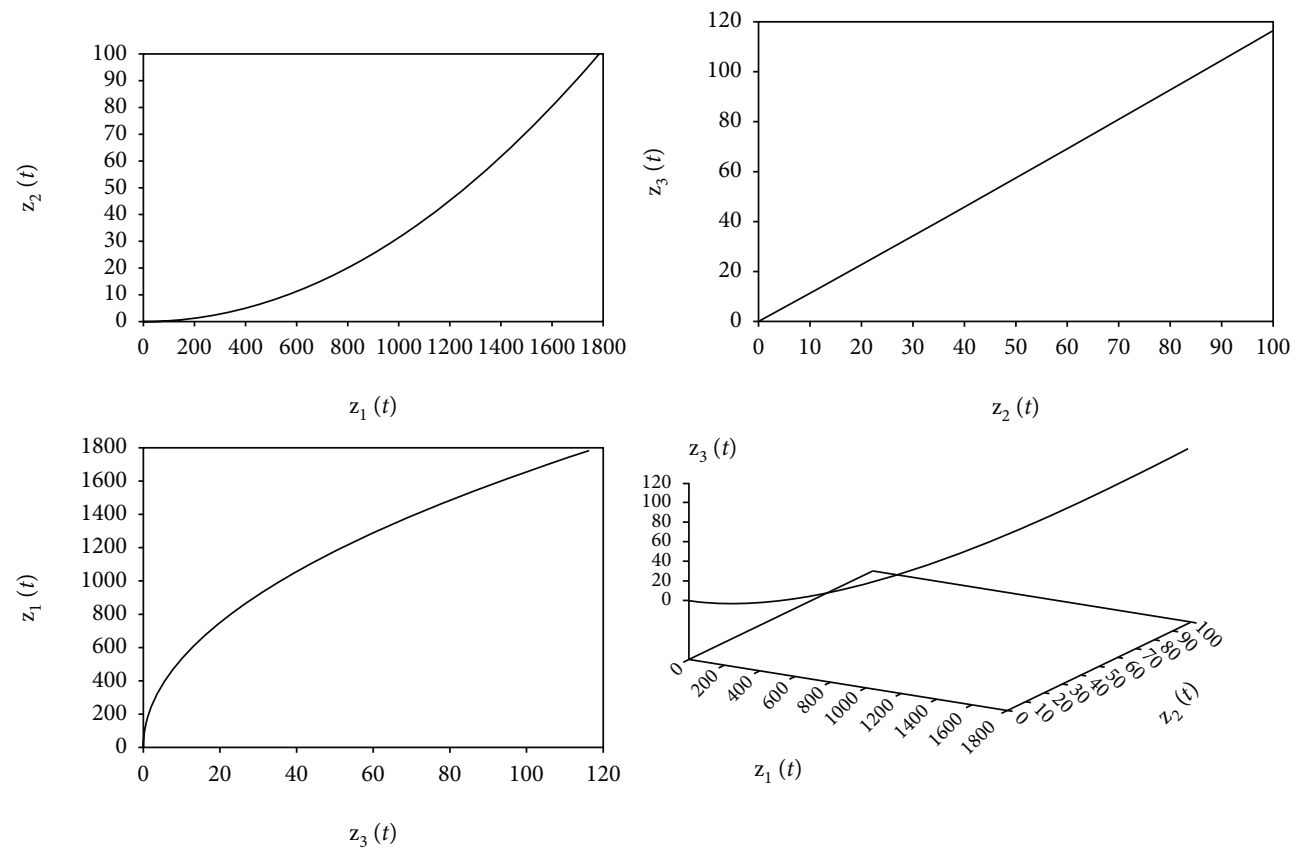

FIGURE 2: Phase portraits using 9-iteration NIM for impulse input. 


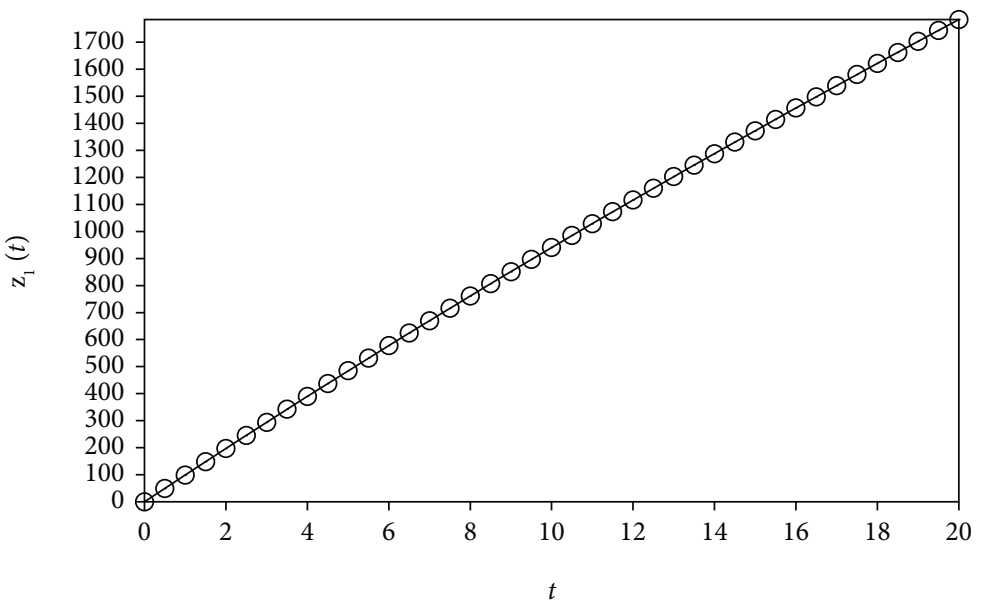

(a)

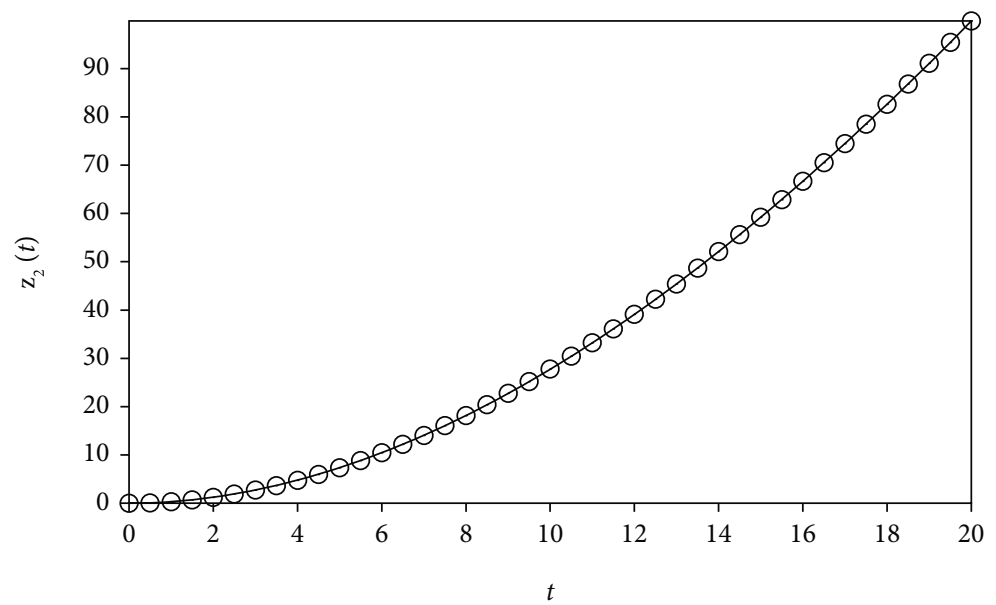

(b)

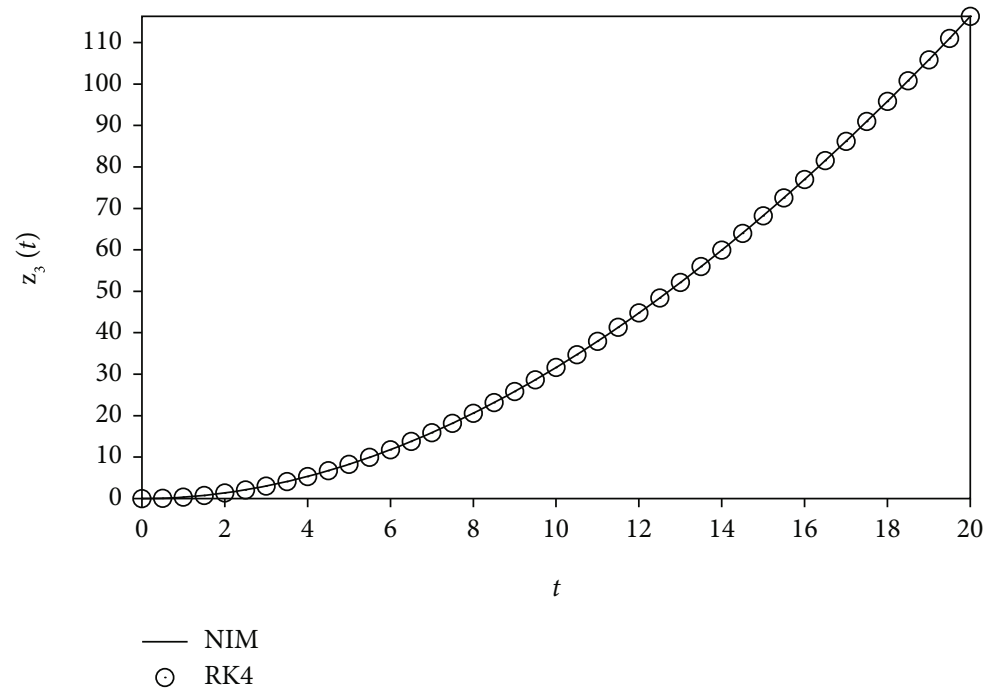

(c)

Figure 3: Comparison among NIM (solid line) and RK4 (circle) for (a) $z_{1}(t)$, (b) $z_{2}(t)$, and (c) $z_{3}(t)$ in the impulse input model. 
TABle 3: Numerical comparisons between ADM and NIM for the step input model.

\begin{tabular}{|c|c|c|c|c|c|c|}
\hline \multirow[b]{2}{*}{$t$} & \multicolumn{3}{|c|}{$\mathrm{ADM}[14]$} & \multicolumn{3}{|c|}{ NIM } \\
\hline & $z_{1}(t)$ & $z_{2}(t)$ & $z_{3}(t)$ & $z_{1}(t)$ & $z_{2}(t)$ & $z_{3}(t)$ \\
\hline 0.01 & 0.00500 & $0.10344 \times 10^{-6}$ & $0.11493 \times 10^{-6}$ & 0.00500 & $0.10344 \times 10^{-6}$ & $0.11493 \times 10^{-6}$ \\
\hline 0.02 & 0.02000 & $0.82744 \times 10^{-6}$ & $0.91942 \times 10^{-6}$ & 0.02000 & $0.82744 \times 10^{-6}$ & $0.91942 \times 10^{-6}$ \\
\hline 0.03 & 0.04499 & $0.27923 \times 10^{-5}$ & $0.31028 \times 10^{-5}$ & 0.04499 & $0.2792 \times 10^{-5}$ & $0.31028 \times 10^{-5}$ \\
\hline 0.04 & 0.07999 & $0.66184 \times 10^{-5}$ & $0.73545 \times 10^{-5}$ & 0.07999 & $0.66184 \times 10^{-5}$ & $0.73544 \times 10^{-5}$ \\
\hline 0.05 & 0.12497 & 0.00001 & 0.00001 & 0.12497 & 0.00001 & 0.00001 \\
\hline 0.06 & 0.17995 & 0.00002 & 0.00002 & 0.17995 & 0.00002 & 0.00002 \\
\hline 0.07 & 0.24493 & 0.00004 & 0.00004 & 0.24493 & 0.00004 & 0.00004 \\
\hline 0.08 & 0.31989 & 0.00005 & 0.00006 & 0.31989 & 0.00005 & 0.00006 \\
\hline 0.09 & 0.40484 & 0.00008 & 0.00008 & 0.40484 & 0.00008 & 0.00008 \\
\hline 0.1 & 0.49978 & 0.00010 & 0.00011 & 0.49978 & 0.00010 & 0.00011 \\
\hline
\end{tabular}

Therefore, the 9-iteration approximate series solutions are

$$
\begin{aligned}
z_{1}(t)= & \sum_{i=0}^{9} z_{1, i}(t)=50 t^{2}-0.2183908046 t^{3} \\
& +0.001640802918 t^{4}-9.157937795 \\
& \times 10^{-6} t^{5}+3.80677017 \times 10^{-10} t^{6}-1.087667026 \\
& \times 10^{-10} t^{7}+1.158119015 \times 10^{-13} t^{8}+8.958217733 \\
& \times 10^{-16} t^{9}-7.447533873 \times 10^{-18} t^{10}+3.557110013 \\
& \times 10^{-20} t^{11} \\
z_{2}(t)= & \sum_{i=0}^{9} z_{2, i}(t)=0.1034482759 t^{3}-0.0008865496258 t^{4} \\
& +5.791657210 \times 10^{-6} t^{5}-2.991487185 \times 10^{-8} t^{6} \\
& +1.242533843 \times 10^{-10} t^{7}-4.132939762 \times 10^{-13} t^{8} \\
& +1.052326691 \times 10^{-15} t^{9}-1.672429216 \times 10^{-18} t^{10} \\
& -9.827203838 \times 10^{-22} t^{11} \\
z_{3}(t)= & \sum_{i=0}^{9} z_{3, i}=0.1149425287 t^{3}-0.0007542532924 t^{4} \\
& +3.366280585 \times 10^{-6} t^{5}-8.152829849 \times 10^{-9} t^{6} \\
& -1.548668175 \times 10^{-11} t^{7}+2.974820747 \times 10^{-13} t^{8} \\
& -1.948148465 \times 10^{-15} t^{9}+9.119963089 \times 10^{-18} t^{10} \\
& -3.458837975 \times 10^{-20} t^{11} .
\end{aligned}
$$

It is easily observed that the obtained solution,

$\left(z_{1}, z_{2}, z_{3}\right)=\left(\sum_{i=0}^{\infty} z_{1, i}, \sum_{i=0}^{\infty} z_{2, i}, \sum_{i=0}^{\infty} z_{3, i}\right)$, converges to the exact solution when

$$
\begin{aligned}
\beta_{1, i} & =\frac{\left\|z_{1, i+1}\right\|}{\left\|z_{1, i}\right\|}<1, \beta_{2, i}=\frac{\left\|z_{2, i+1}\right\|}{\left\|z_{2, i}\right\|}<1, \beta_{3, i} \\
& =\frac{\left\|z_{3, i+1}\right\|}{\left\|z_{3, i}\right\|}<1, \text { for } i \geq 1,0<t \leq 1 .
\end{aligned}
$$

TABLE 4: Differences between 9-iteration NIM with RK4 solutions in the step input model.

\begin{tabular}{lccc}
\hline & & $\Delta=\left|R K 4_{0.001}-\mathrm{NIM}\right|$ & \\
$\boldsymbol{t}$ & $\Delta z_{1}(t)$ & $\Delta z_{2}(t)$ & $\Delta z_{3}(t)$ \\
\hline 2 & $1.60 e-11$ & $1.203 e-13$ & $1.365 e-13$ \\
4 & $8.11 e-11$ & $1.265 e-12$ & $1.421 e-12$ \\
6 & $2.45 e-10$ & $4.48 e-12$ & $4.88 e-12$ \\
8 & $6.81 e-10$ & $1.780 e-11$ & $1.424 e-11$ \\
10 & $1.50 e-10$ & $2.311 e-11$ & $1.127 e-10$ \\
12 & $2.81 e-10$ & $2.196 e-10$ & $1.019 e-09$ \\
14 & $4.476 e-09$ & $1.191 e-09$ & $6.223 e-09$ \\
16 & $3.119 e-08$ & $5.482 e-09$ & $3.029 e-08$ \\
18 & $1.439 e-07$ & $2.148 e-08$ & $1.228 e-07$ \\
20 & $5.113 e-07$ & $7.496 e-08$ & $4.322 e-07$ \\
\hline
\end{tabular}

Here, we can see that

$$
\begin{aligned}
& \frac{\left\|z_{1,2}\right\|}{\left\|z_{1,1}\right\|}=\frac{\left\|\left(16283 t^{3}\right) / 9923800\right\|}{\left\|-\left(19 t^{2}\right) / 87\right\|}=0.00751315016<1, \\
& \frac{\left\|z_{2,2}\right\|}{\left\|z_{2,1}\right\|}=\frac{\left\|-\left(507 t^{3}\right) / 571880\right\|}{\left\|\left(3 t^{2}\right) / 29\right\|}=0.02916446054<1, \\
& \frac{\left\|z_{3,2}\right\|}{\left\|z_{3,1}\right\|}=\frac{\left\|-\left(63623 t^{3}\right) / 84352300\right\|}{\left\|\left(10 t^{2}\right) / 87\right\|}=0.00656200362<1 .
\end{aligned}
$$

This confirms that the NIM series solution converges to the exact solution.

4.3. Sinusoidal Input. When concentration of the lake converges to the average input concentration of the contaminant [14], then the input is periodic input, and we assume $q(t)=1+\sin (t)$

$$
\frac{d z_{1}}{d t}=\frac{38}{1180} z_{3}(t)+\{1+\sin (t)\}-\frac{38}{2900} z_{1}(t)
$$




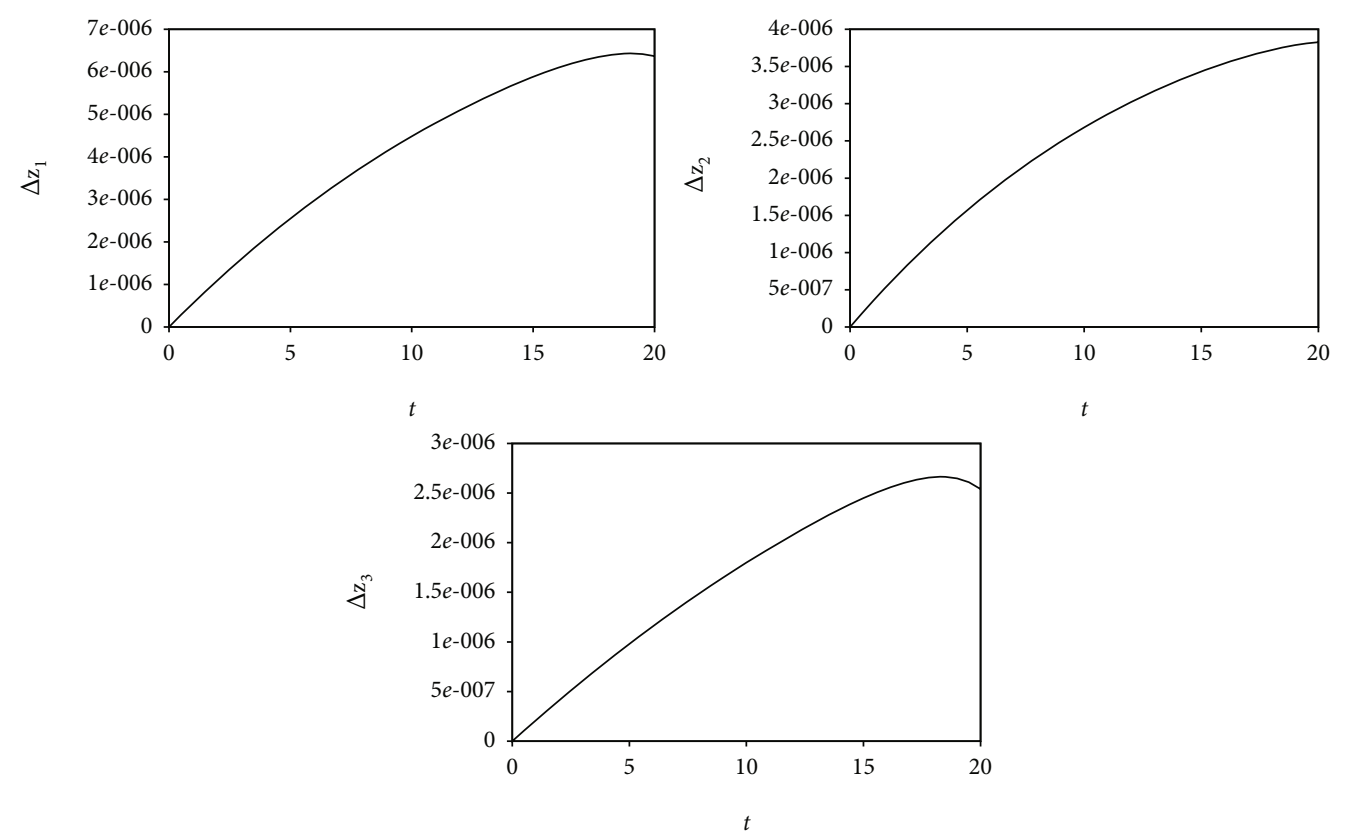

Figure 4: Absolute error (A.E.) of step input for $\Delta z_{1}(t), \Delta z_{2}(t)$, and $\Delta z_{3}(t)$.

$$
\begin{aligned}
& \frac{d z_{2}}{d t}=\frac{18}{2900} z_{1}(t)-\frac{18}{850} z_{2}(t), \\
& \frac{d z_{3}}{d t}=\frac{20}{2900} z_{1}(t)+\frac{18}{850} z_{2}(t)-\frac{38}{1180} z_{3}(t),
\end{aligned}
$$

with initial conditions $z_{1}(0)=z_{2}(0)=z_{3}(0)=0$.

Integrating the system of equations from 0 to $t$ and using the initial conditions (51), we get

$$
\begin{aligned}
& z_{1}(t)=1+t-\cos (t)+\int_{0}^{t} \frac{19 z_{3}(t)}{590} d t-\int_{0}^{t} \frac{19 z_{1}(t)}{1450} d t, \\
& z_{2}(t)=\int_{0}^{t} \frac{9 z_{1}(t)}{1450} d t-\int_{0}^{t} \frac{9 z_{2}(t)}{425} d t, \\
& z_{3}(t)=\int_{0}^{t} \frac{z_{1}(t)}{145} d t+\int_{0}^{t} \frac{9 z_{2}(t)}{425} d t-\int_{0}^{t} \frac{19 z_{3}(t)}{590} d t .
\end{aligned}
$$

We let the nonlinear terms as

$$
\begin{aligned}
& N_{1}\left(z_{1}, z_{2}, z_{3}\right)=\int_{0}^{t} \frac{19 z_{3}(t)}{590} d t-\int_{0}^{t} \frac{19 z_{1}(t)}{1450} d t, \\
& N_{2}\left(z_{1}, z_{2}, z_{3}\right)=\int_{0}^{t} \frac{9 z_{1}(t)}{1450} d t-\int_{0}^{t} \frac{9 z_{2}(t)}{425} d t, \\
& N_{3}\left(z_{1}, z_{2}, z_{3}\right)=\int_{0}^{t} \frac{z_{1}(t)}{145} d t+\int_{0}^{t} \frac{9 z_{2}(t)}{425} d t-\int_{0}^{t} \frac{19 z_{3}(t)}{590} d t .
\end{aligned}
$$

In view of (11)-(14), we have the following first few components of the new iterative solution for the considered problem:

$$
z_{1,0}(t)=1+t-\cos (t)
$$

$$
z_{2,0}(t)=0,(63)
$$

$$
\begin{aligned}
z_{3,0}(t)= & 0, \\
z_{1,1}(\mathrm{t})= & N_{1}\left(z_{1,0}, z_{2,0}, z_{3,0}\right)=\int_{0}^{t} \frac{19 z_{3,0}(t)}{590} d t-\int_{0}^{t} \frac{19 z_{1,0}(t)}{1450} d t \\
= & -\frac{19 t}{1450}-\frac{19 t^{2}}{2900}+\frac{19 \sin (t)}{1450}, \\
z_{2,1}(t)= & N_{2}\left(z_{1,0}, z_{2,0}, z_{3,0}\right)=\int_{0}^{t} \frac{9 z_{1,0}(t)}{1450} d t-\int_{0}^{t} \frac{9 z_{2,0}(t)}{425} d t \\
= & \frac{9 t}{1450}+\frac{9 t^{2}}{2900}-\frac{9 \sin (t)}{1450}, \\
z_{3,1}(t)= & N_{3}\left(z_{1,0}, z_{2,0}, z_{3,0}\right)=\int_{0}^{t} \frac{z_{1,0}(t)}{145} d t+\int_{0}^{t} \frac{9 z_{2,0}(t)}{425} d t \\
& -\int_{0}^{t} \frac{19 z_{3,0}(t)}{590} d t=\frac{t}{145}+\frac{t^{2}}{290}-\frac{\sin (t)}{145}, \\
z_{1,2}(t)= & N_{1}\left(z_{1,0}+z_{1,1}, z_{2,0}+z_{2,1}, z_{3,0}+z_{3,1}\right)-N_{1}\left(z_{1,0}, z_{2,0}, z_{3,0}\right) \\
= & \left\{\int_{0}^{t} \frac{19\left(z_{3,0}(t)+z_{3,1}(t)\right)}{590} d t-\int_{0}^{t} \frac{19\left(z_{1,0}(t)+z_{1,1}(t)\right)}{1450} d t\right\} \\
& -\left\{\int_{0}^{t} \frac{19 z_{3,0}(t)}{590} d t-\int_{0}^{t} \frac{19 z_{1,0}(t)}{1450} d t\right\} \\
= & -\frac{48849}{124047500}+\frac{48849 t^{2}}{248095000}+\frac{16283 t^{3}}{248095000}+\frac{48849 \cos (t)}{124047500},
\end{aligned}
$$




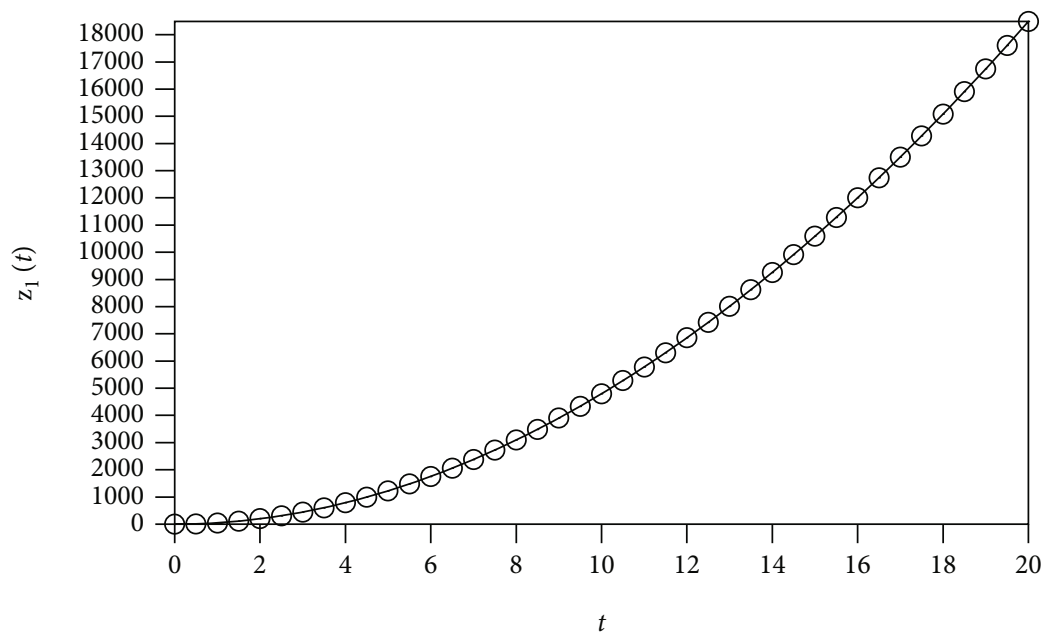

(a)

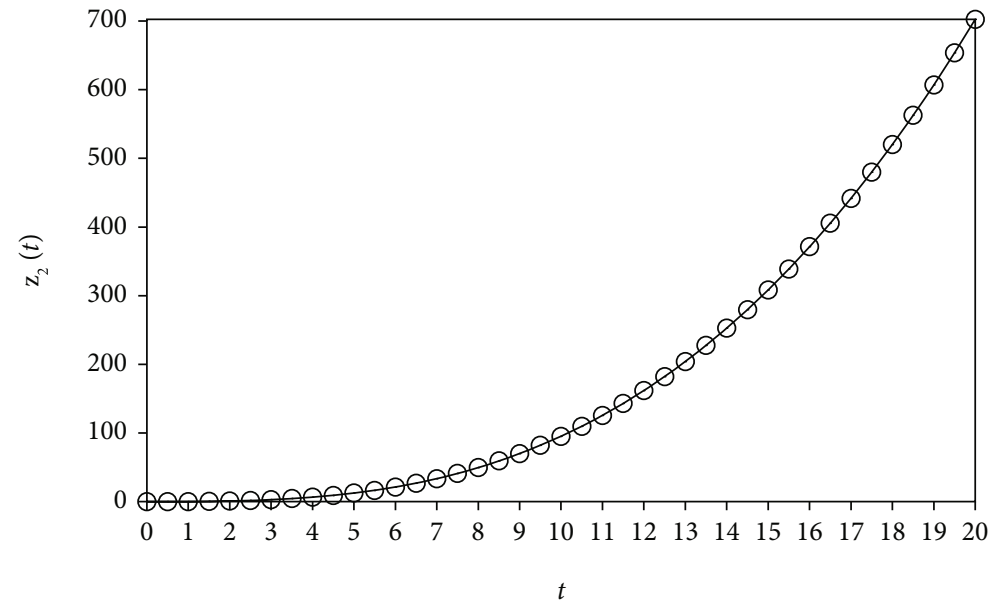

(b)

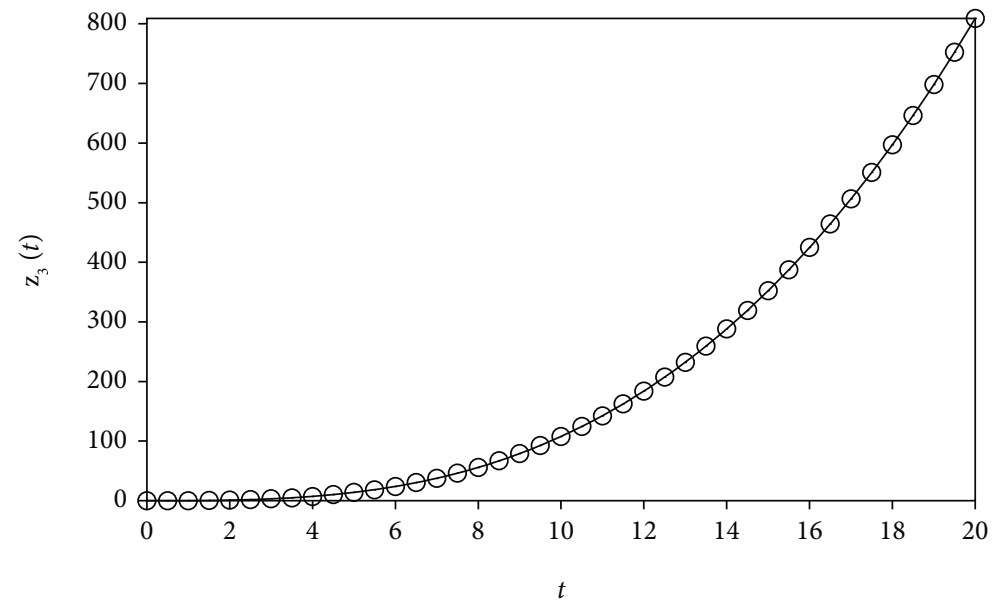

- NIM

○ RK4

(c)

Figure 5: Comparison among NIM (solid line) and RK4 (circle) for (a) $z_{1}(t)$, (b) $z_{2}(t)$, and (c) $z_{3}(t)$ in the step input model. 
TABLE 5: Numerical comparisons between ADM and NIM for the sinusoidal input model.

\begin{tabular}{|c|c|c|c|c|c|c|}
\hline \multirow[b]{2}{*}{$t$} & \multicolumn{3}{|c|}{ ADM [14] } & \multicolumn{3}{|c|}{ NIM } \\
\hline & $z_{1}(t)$ & $z_{2}(t)$ & $z_{3}(t)$ & $z_{1}(t)$ & $z_{2}(t)$ & $z_{3}(t)$ \\
\hline 0.01 & 0.01005 & $0.31134 \times 10^{-6}$ & $0.34595 \times 10^{-6}$ & 0.01005 & $0.31134 \times 10^{-6}$ & $0.34595 \times 10^{-6}$ \\
\hline 0.02 & 0.02020 & $0.12494 \times 10^{-5}$ & $0.13883 \times 10^{-5}$ & 0.02020 & $0.12494 \times 10^{-5}$ & $0.13883 \times 10^{-5}$ \\
\hline 0.03 & 0.03044 & $0.28201 \times 10^{-5}$ & $0.31337 \times 10^{-5}$ & 0.03044 & $0.28201 \times 10^{-5}$ & $0.31337 \times 10^{-5}$ \\
\hline 0.04 & 0.04079 & $0.50294 \times 10^{-5}$ & $0.55888 \times 10^{-5}$ & 0.04079 & $0.50294 \times 10^{-5}$ & $0.55888 \times 10^{-5}$ \\
\hline 0.05 & 0.05123 & $0.78834 \times 10^{-5}$ & $0.87605 \times 10^{-5}$ & 0.05123 & $0.78834 \times 10^{-5}$ & $0.87605 \times 10^{-5}$ \\
\hline 0.06 & 0.06178 & 0.00001 & 0.00001 & 0.06178 & 0.00001 & 0.00001 \\
\hline 0.07 & 0.07242 & 0.00002 & 0.00002 & 0.07242 & 0.00002 & 0.00002 \\
\hline 0.08 & 0.08316 & 0.00002 & 0.00002 & 0.08316 & 0.00002 & 0.00002 \\
\hline 0.09 & 0.09399 & 0.00002 & 0.00003 & 0.09399 & 0.00003 & 0.00003 \\
\hline 0.1 & 0.10493 & 0.00003 & 0.00004 & 0.10493 & 0.00003 & 0.00004 \\
\hline
\end{tabular}

TABLE 6: Differences between 9-iteration NIM with RK4 solutions in the sinusoidal input model.

\begin{tabular}{lccc}
\hline & \multicolumn{3}{c}{$\Delta=\left|R K 4_{0.001}-\mathrm{NIM}\right|$} \\
$t$ & $\Delta z_{1}(t)$ & $\Delta z_{2}(t)$ & $\Delta z_{3}(t)$ \\
\hline 2 & $1.3 e-14$ & 0 & $2 e-16$ \\
4 & $9 e-15$ & $2 e-16$ & $5 e-16$ \\
6 & $1.0 e-14$ & $2 e-16$ & $8.4 e-15$ \\
8 & $1.85 e-13$ & $2.77 e-14$ & $1.690 e-13$ \\
10 & $2.33 e-12$ & $3.479 e-13$ & $1.974 e-12$ \\
12 & $1.726 e-11$ & $2.572 e-12$ & $1.462 e-11$ \\
14 & $9.251 e-11$ & $1.378 e-11$ & $7.863 e-11$ \\
16 & $3.933 e-10$ & $5.847 e-11$ & $3.349 e-10$ \\
18 & $1.404 e-09$ & $2.079 e-10$ & $1.196 e-09$ \\
20 & $4.366 e-09$ & $6.444 e-10$ & $3.722 e-09$ \\
\hline
\end{tabular}

$$
\begin{aligned}
z_{2,2}(t)= & N_{2}\left(z_{1,0}+z_{1,1}, z_{2,0}+z_{2,1}, z_{3,0}+z_{3,1}\right)-N_{1}\left(z_{1,0}, z_{2,0}, z_{3,0}\right) \\
= & \left\{\int_{0}^{t} \frac{9\left(z_{1,0}(t)+z_{1,1}(t)\right)}{1450} d t-\int_{0}^{t} \frac{9\left(z_{2,0}(t)+z_{2,1}(t)\right)}{425} d t\right\} \\
& -\left\{\int_{0}^{t} \frac{9 z_{1,0}(t)}{1450} d t-\int_{0}^{t} \frac{9 z_{2,0}(t)}{425} d t\right\}=\frac{1521}{7148500} \\
& -\frac{1521 t^{2}}{14297000}-\frac{507 t^{3}}{14297000}-\frac{1521 \cos (t)}{7148500}, \\
z_{3,2}(t)= & N_{3}\left(z_{1,0}+z_{1,1}, z_{2,0}+z_{2,1}, z_{3,0}+z_{3,1}\right)-N_{1}\left(z_{1,0}, z_{2,0}, z_{3,0}\right) \\
= & \left\{\int_{0}^{t} \frac{\left(z_{1,0}(t)+z_{1,1}(t)\right)}{145} d t+\int_{0}^{t} \frac{9\left(z_{2,0}(t)+z_{2,1}(t)\right)}{425} d t\right. \\
& \left.-\int_{0}^{t} \frac{19\left(z_{3,0}(t)+z_{3,1}(t)\right)}{590} d t\right\}-\left\{\int_{0}^{t} \frac{z_{1,0}(t)}{145} d t\right. \\
& \left.+\int_{0}^{t} \frac{9 z_{2,0}(t)}{425} d t-\int_{0}^{t} \frac{19 z_{3,0}(t)}{590} d t\right\}=\frac{190869}{1054403750} \\
& -\frac{190869 t^{2}}{2108807500}-\frac{63623 t^{3}}{2108807500}-\frac{190869 \cos (t)}{1054403750},
\end{aligned}
$$

and so on. In the same manner, the rest of components can be obtained.
Therefore, the 9-iteration approximate series solutions are

$$
\begin{aligned}
z_{1}(t)= & \sum_{i=0}^{9} z_{1, i}(t)=0.01309246424 \sin (t)-7.548439160 \\
& \times 10^{-12} t^{6}+0.9869075358 t+7.392117911 \\
& \times 10^{-17} t^{8}+2.238353115 \times 10^{-9} t^{5}-4.464785362 \\
& \times 10^{-7} t^{4}+9.907126563 \times 10^{-15} t^{7} \\
& +0.00006380144335 \times t^{3}-0.006354964808 \\
& \times t^{2}-0.9996064813 \cos (t)+0.9996064813 \\
& +3.912821015 \times 10^{-21} t^{10}-7.056251772 \times 10^{-19} t^{9},
\end{aligned}
$$$$
z_{2}(t)=\sum_{i=0}^{9} z_{2, i}(t)=-0.006199952822 \sin (t)
$$$$
+8.466376566 \times 10^{-12} t^{6}+0.006199952822 t
$$$$
+9.320421594 \times 10^{-17} t^{8}-1.742737715 \times 10^{-9} t^{5}
$$$$
+2.806153398 \times 10^{-7} t^{4}-3.230576505 \times 10^{-14} t^{7}
$$$$
+0.0002125566897-0.00003430469668 t^{3}
$$$$
+0.002997169931 t^{2}-0.0002125566897 \cos (t)
$$$$
-1.080992422 \times 10^{-22} t^{10}-1.683239140 \times 10^{-19} t^{9} \text {, }
$$

$$
\begin{aligned}
z_{3}(t)= & \sum_{i=0}^{9} z_{3, i}=-0.006892511414 \sin (t) \\
& -9.179374067 \times 10^{-13} t^{6}+0.006892511414 t \\
& -1.671253951 \times 10^{-16} t^{8}-4.956154003 \\
& \times 10^{-10} t^{5}+1.658631964 \times 10^{-7} t^{4}+2.239863848 \\
& \times 10^{-14} t^{7}-0.00002949674667 t^{3} \\
& +0.003357794877 t^{2}-0.0001809619702 \cos (t) \\
& -3.804721773 \times 10^{-21} t^{10}+8.739490912 \times 10^{-19} t^{9} \\
& +0.0001809619702
\end{aligned}
$$



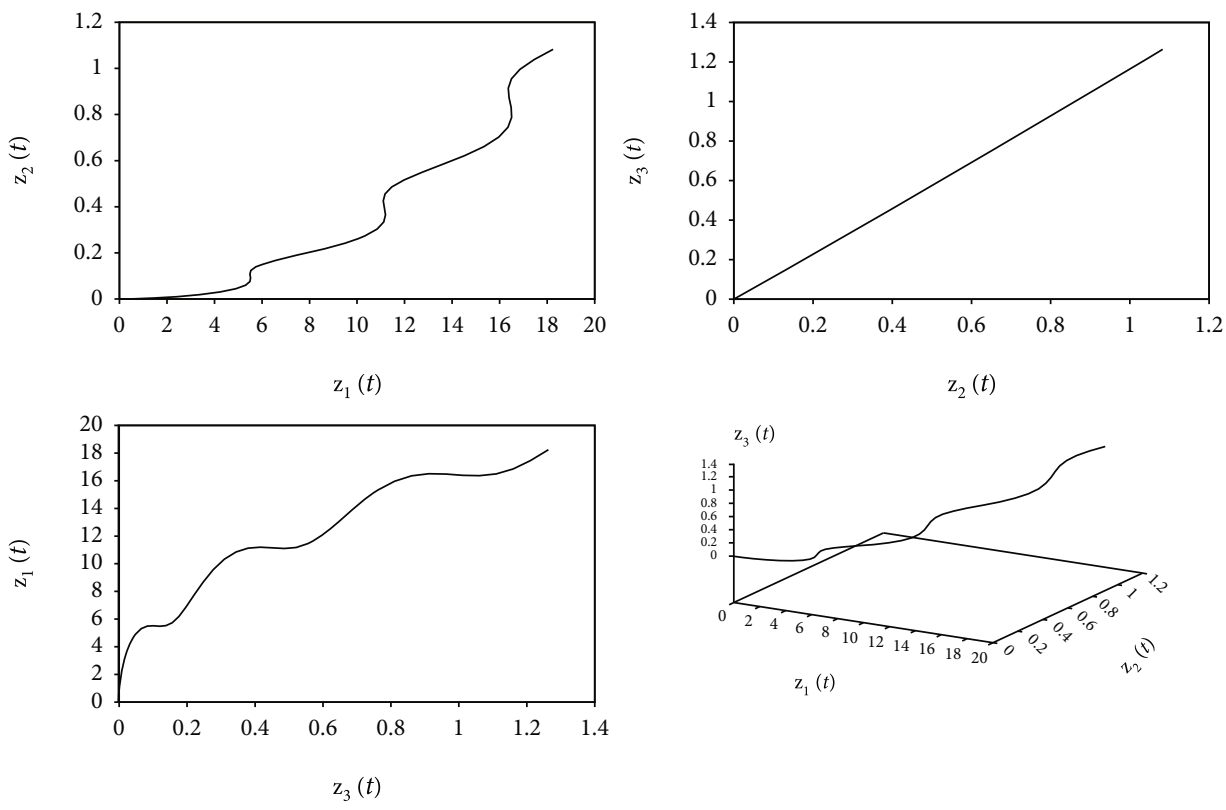

FIGURE 6: Phase portraits using 9-iteration NIM for sinusoidal input.

It is easily observed that the obtained solution, $\left(z_{1}, z_{2}\right.$, $\left.z_{3}\right)=\left(\sum_{i=0}^{\infty} z_{1, i}, \sum_{i=0}^{\infty} z_{2, i}, \sum_{i=0}^{\infty} z_{3, i}\right)$, converges to the exact solution when

$\beta_{1, i}=\frac{\left\|z_{1, i+1}\right\|}{\left\|z_{1, i}\right\|}<1, \beta_{2, i}=\frac{\left\|z_{2, i+1}\right\|}{\left\|z_{2, i}\right\|}<1, \beta_{3, i}=\frac{\left\|z_{3, i+1}\right\|}{\left\|z_{3, i}\right\|}<1$,

for $i \geq 1,0<t \leq 1$.

Here, we can see that

$\frac{\left\|z_{1,2}\right\|}{\left\|z_{1,1}\right\|}=\frac{\left\|-48849 / 124047500+\left(48849 t^{2}\right) / 248095000+\left(16283 t^{3}\right) / 248095000+(48849 \cos (t)) / 124047500\right\|}{\left\|-19 t / 1450-\left(19 t^{2}\right) / 2900+(19 \sin (t)) / 1450\right\|}=0.00944522675<1$,
$\frac{\left\|z_{2,2}\right\|}{\left\|z_{2,1}\right\|}=\frac{\left\|1521 / 7148500-\left(1521 t^{2}\right) / 14297000-\left(507 t^{3}\right) / 14297000-(1521 \cos (t)) / 7148500\right\|}{\left\|9 t / 1450+\left(9 t^{2}\right) / 2900-(9 \sin (t)) / 1450\right\|}=0.010773829<1$,
$\frac{\left\|z_{3,2}\right\|}{\left\|z_{3,1}\right\|}=\frac{\left\|190869 / 1054403750-\left(190869 t^{2}\right) / 2108807500-\left(63623 t^{3}\right) / 2108807500-(190869 \cos (t)) / 1054403750\right\|}{\left\|t / 145+t^{2} / 290-\sin (t) / 145\right\|}=0.00824948252<1$.

This confirms that the NIM series solution converges to the exact solution.

\section{Results and Discussions}

In this work, we have presented the new iterative method (NIM) as a useful analytic-numeric tool to solve pollution model for a system of three interconnecting lakes. The NIM algorithm is coded in the computer algebra package Maple software. The Maple condition variable digits controlling the number of significant digits are set to 16 in all the calculations done in this paper. We additionally fix the estimations of the parameters and take the initial conditions as stated in [14]. The time domain studied in this work is $[0,20]$, same as considered in [20]. For comparison purposes, the fourth-order Runge-Kutta algorithm (RK4) build in Maple software was used to obtain the numeric solution of the pollution problems.

5.1. Impulse Input. In Table 1, we have made tabular comparison between numeric solutions of NIM and ADM for the impulse input model for $t$ up to 0.1 only to directly compare with [17]. The 9-iteration NIM solutions agree with the RK4 solutions at least 7 decimal places as shown in Table 2 for 


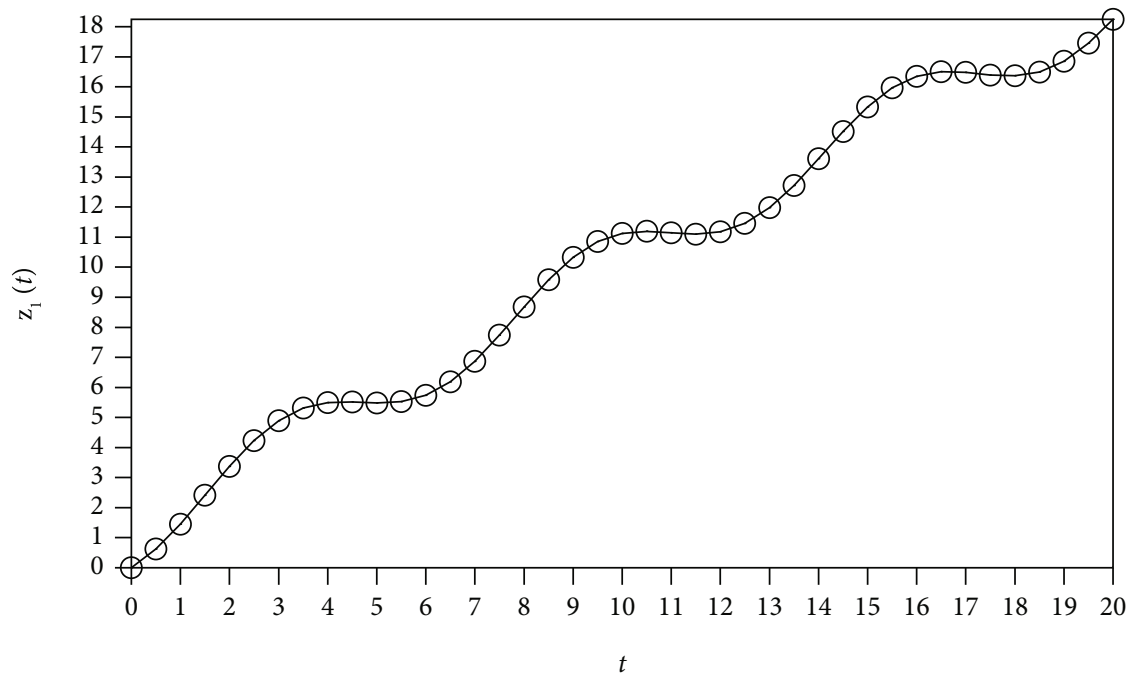

(a)

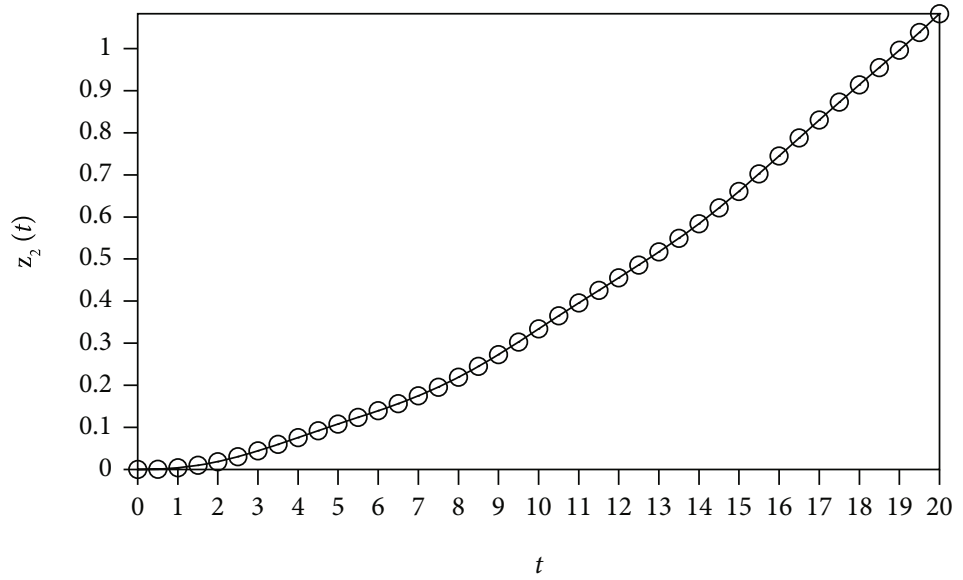

(b)

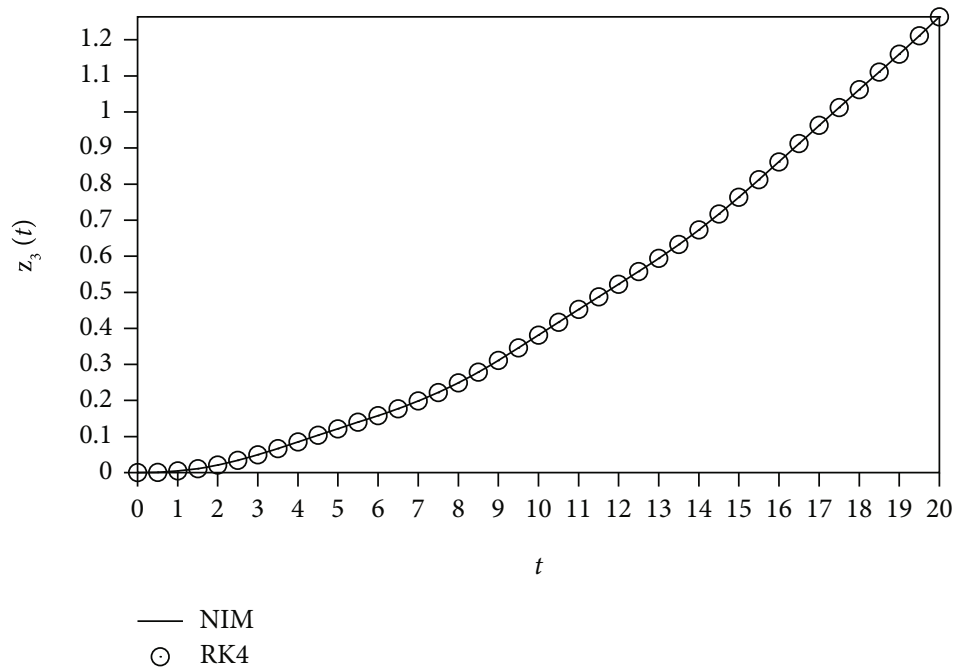

(c)

FIGURE 7: Comparison among 9-iteration NIM (solid line) and RK4 (circle) for (a) $z_{1}(t),(\mathrm{b}) z_{2}(t)$, and (c) $z_{3}(t)$ in the sinusoidal input model.

impulse input for $t$ up to 20. We have figured out the $z_{1}-z_{2}, z_{2}$ $-z_{3}, z_{1}-z_{3}$, and $z_{1}-z_{2}-z_{3}$ phase portraits of 9-iteration NIM for the impulse input system in Figure 2 which shows their smooth behavior in the phase plane. Figure 3 shows the graphical comparison among RK4 solutions and the NIM approximations for the impulse input models for $t$ up to 20 . 
5.2. Step Input. In Table 3 , we have made tabular comparison between numeric solutions of NIM and ADM for the step input model for $t$ up to 0.1 only as [17]. We have noted from Table 4 that the NIM precisions match to those of RK4 at least 6 decimal places for $t$ up to 20. The routine was configured to find out the graphical representation of absolute errors (A.E.) for the step input model as it can be seen in Figure 4. In general terms, the absolute error is low for all approximations. If required, the error can be further reduced by increasing the iterations of the NIM approximations. Figure 5 shows the graphical comparison among RK4 solutions and the NIM approximations for the step input models for $t$ up to 20 .

5.3. Sinusoidal Input. In Table 5, we have made tabular comparison between numeric solutions of NIM and ADM [17] for the sinusoidal input model for $t$ up to 0.1 same as [17]. Similarly, we have noticed from Table 6 that the NIM precisions match to those of RK4 at least minimum 5 decimal places for sinusoidal inputs for $t$ up to 20 . We reproduced the $z_{1}-z_{2}, z_{2}-z_{3}, z_{1}-z_{3}$, and $z_{1}-z_{2}-z_{3}$ phase portraits of 9-iteration NIM for the sinusoidal input system in Figure 6 which shows their smooth behavior in the phase plane. Figure 7 shows the graphical comparison among RK4 solutions and the NIM approximations for the sinusoidal input models for $t$ up to 20 .

All of the above discussions indicate that NIM is very accurate, requires less computation work, very easy to implement, and consumes less time to obtain good approximate solution in a wide interval for lake pollution problem.

\section{Conclusions}

In this study, the new iterative method (NIM) has been successfully implemented to gain the approximate analytic as well as numeric solutions of the lake pollution model and results compared with the traditional fourth-order Runge Kutta method (RK4) and ADM. The potential advantages of NIM are summarized below:

(a) This comparison shows that the solution procedure of the NIM is simpler than of ADM, LPDTM without any special type of polynomials or multipliers. Simple solution procedure, high accuracy and efficiency in computation are the advantages of the proposed method

(b) The accuracy of graphical and numerical results of NIM is very high in large time domain

(c) There are difficulties arising in calculating Adomian polynomials to handle the non-linear terms in ADM. It is necessary to calculate Lagrange multiplier in VIM in which the terms of the sequence become complex after several iterations; thus, finding more iterations in VIM becomes more laborious. Also, there is a need to construct a homotopy parameter in HPM and solving the corresponding algebraic equations will be somehow difficult. The advantages of NIM over other iterative methods (such as ADM,
VIM, HPM) are its very straightforward in computer programming with less computation works and at the same time it provides fast convergent analyticnumeric solutions without any transformation, linearization, and discretization

The outcomes introduced in this paper reveal that NIM is promptly pertinent to systems of ODEs including more complex physical models.

\section{Data Availability}

The data used in the article is generated by Maple program that can be made available upon request.

\section{Conflicts of Interest}

The authors declare that they have no conflicts of interest.

\section{Acknowledgments}

The authors are grateful for the monetary help from the Ministry of Higher Education and International Islamic University Malaysia via the FRGS-RACER Grant no. RACER/1/2019/TK10/UIAM//1.

\section{References}

[1] S. M. Aznam, N. A. Che Ghani, and M. S. H. Chowdhury, "A numerical solution for nonlinear heat transfer of fin problems using the Haar wavelet quasilinearization method," Results in Physics, vol. 14, p. 102393, 2019.

[2] S. Peiravi, "Numerical study of fins arrangement and nanofluids effects on three-dimensional natural convection in the cubical enclosure," ransport Phenomena in Nano and Micro Scales, vol. 7, no. 2, pp. 97-112, 2019.

[3] S. Mungkasi, "Variational iteration and successive approximation methods for a SIR epidemic model with constant vaccination strategy," Applied Mathematical Modelling, vol. 90, pp. 1-10, 2021.

[4] J. Alinejad and M. M. Peiravi, "Numerical analysis of secondary droplets characteristics due to drop impacting on 3D cylinders considering dynamic contact angle," Meccanica, vol. 55, no. 10, pp. 1975-2002, 2020.

[5] M. M. Peiravi and J. Alinejad, "Hybrid conduction, convection and radiation heat transfer simulation in a channel with rectangular cylinder," Journal of Thermal Analysis and Calorimetry, vol. 140, no. 6, pp. 2733-2747, 2020.

[6] M. M. Peiravi, J. Alinejad, D. D. Ganji, and S. Maddah, “3D optimization of baffle arrangement in a multi-phase nanofluid natural convection based on numerical simulation," International Journal of Numerical Methods for Heat and Fluid Flow, vol. 30, no. 5, pp. 2583-2605, 2019.

[7] M. A. Hosen and M. S. H. Chowdhury, "A new analytical technique based on harmonic balance method to determine approximate periods for Duffing-harmonic oscillator," Alexandria Engineering Journal, vol. 54, no. 2, pp. 233-239, 2015.

[8] S. M. Aznam and M. S. H. Chowdhury, "Generalized Haar wavelet operational matrix method for solving hyperbolic heat conduction in thin surface layers," Results in Physics, vol. 11, pp. 243-252, 2018. 
[9] M. Madani, M. Fathizadeh, Y. Khan, and A. Yildirim, "On the coupling of the homotopy perturbation method and Laplace transformation," Mathematical and Computer Modelling, vol. 53, no. 9-10, pp. 1937-1945, 2011.

[10] M. S. H. Chowdhury and I. Hashim, "Application of homotopy-perturbation method to Klein-Gordon and sineGordon equations," Chaos, Solitons and Fractals, vol. 39, no. 4, pp. 1928-1935, 2009.

[11] N. I. Razali, M. S. H. Chowdhury, and W. Asrar, "The multistage adomian decomposition method for solving chaotic lü system," Middle-East Journal of Scientific Research, vol. 13, pp. 43-49, 2013.

[12] M. S. H. Chowdhury, N. I. Razali, S. Ali, and M. M. Rahman, "An accurate analytic solution for differential and integral equations by modified homotopy perturbation method," Middle-East Journal of Scientific Research, vol. 13, pp. 50-58, 2013.

[13] D. D. Ganji, M. Jannatabadi, and E. Mohseni, "Application of He's variational iteration method to nonlinear Jaulent-Miodek equations and comparing it with ADM," Journal of Computational and Applied Mathematics, vol. 207, no. 1, pp. 35-45, 2007.

[14] J. Biazar, L. Farrokhi, and M. R. Islam, "Modeling the pollution of a system of lakes," Applied Mathematics and Computation, vol. 178, no. 2, pp. 423-430, 2006.

[15] D. D. Ganji, M. G. Bandpy, and M. Mostofi, "Simulation of lakes pollution distribution by homotopy perturbation method," International Journal on Engineering Applications (IREA), vol. 6, no. 2, pp. 52-56, 2018.

[16] M. M. El-Dessoky Ahmed and M. Altaf Khan, "Modeling and analysis of the polluted lakes system with various fractional approaches," Chaos, Solitons \& Fractals, vol. 134, p. 109720, 2020.

[17] Ş. Yüzbaşi, N. Şahin, and M. Sezer, “A collocation approach to solving the model of pollution for a system of lakes," Mathematical and Computer Modelling, vol. 55, no. 3-4, pp. 330$341,2012$.

[18] V. Fuat Hatipoğlu, "A novel model for the contamination of a system of three artificial lakes," Discrete \& Continuous Dynamical Systems Series S, 2018.

[19] J. Biazar and T. Rahimi, "Differential transform method for the solution of the lake pollution problem," Natural Sciences, vol. 2013, pp. 103-113, 2013.

[20] B. Benhammouda, H. Vazquez-Leal, and L. Hernandez-Martinez, "Modified differential transform method for solving the model of pollution for a system of lakes," Discrete Dynamics in Nature and Society, vol. 2014, Article ID 645726, 12 pages, 2014.

[21] J. Biazar, M. Shahbala, and H. Ebrahimi, "VIM for solving the pollution problem of a system of lakes," Journal of Control Science and Engineering, vol. 2010, Article ID 829152, 6 pages, 2010.

[22] V. Daftardar-Gejji and H. Jafari, "An iterative method for solving nonlinear functional equations," Journal of Mathematical Analysis and Applications, vol. 316, no. 2, pp. 753-763, 2006.

[23] A. A. Hemeda and E. E. Eladdad, "ew iterative methods for solving Fokker-Planck equation," Mathematical Problems in Engineering, vol. 2018, 2013.

[24] M. S. H. Chowdhury, I. Ghosh, S. M. T. Aznam, and S. Mawa, "A novel iterative method for solving chemical kinetics system," Journal of Low Frequency Noise, Vibration and Active Control, pp. 1-13, 2021.
[25] A. A. Alderremy, T. M. Elzaki, and M. Chamekh, "New transform iterative method for solving some Klein-Gordon equations," Results in Physics, vol. 10, pp. 655-659, 2018.

[26] A. A. Hemeda, "New iterative method: an application for solving fractional physical differential equations," Abstract and Applied Analysis, vol. 1, no. 2, 2013.

[27] M. A. Al-Jawary, M. I. Adwan, and G. H. Radhi, “Three iterative methods for solving second order nonlinear ODEs arising in physics," Journal of King Saud University - Science, vol. 32, no. 1, pp. 312-323, 2020.

[28] M. A. AL-Jawary and O. M. Salih, "Reliable iterative methods for 1D Swift-Hohenberg equation," Arab Journal of Basic and Applied Sciences, vol. 27, no. 1, pp. 56-66, 2020.

[29] M. I. Adwan, M. A. Al-Jawary, J. Tibaut, and J. Ravnik, “Analytic and numerical solutions for linear and nonlinear multidimensional wave equations," Arab Journal of Basic and Applied Sciences, vol. 27, no. 1, pp. 166-182, 2020.

[30] J. Aguirre and D. Tully, Lake Pollution Model, pp. 1-10, 1999, https://mse.redwoods.edu/darnold/math55/DEProj/Sp99/ DarJoel/lakepollution.pdf.

[31] M. A. Noor, M. Waseem, and K. I. Noor, "New iterative technique for solving a system of nonlinear equations," Applied Mathematics and Computation, vol. 271, pp. 446-466, 2015.

[32] S. Bhalekar and V. Daftardar-Gejji, "New iterative method: application to partial differential equations," Applied Mathematics and Computation, vol. 203, no. 2, pp. 778-783, 2008.

[33] M. C. Joshi and R. K. Bose, Some Topics in Nonlinear Functional Analysis, Wiley, 1985.

[34] S. Bhalekar and V. Daftardar-Gejji, "Convergence of the new iterative method," International Journal of Differential Equations, vol. 2011, Article ID 989065, 10 pages, 2011.

[35] A. Jerri, Introduction to Integral Equations with Applications, Wiley, 1999. 